The University of San Francisco

USF Scholarship: a digital repository @ Gleeson Library |

Geschke Center

6-1-1995

\title{
Electron Transport in Magnetic Inhomogeneous Media
}

Horacio E. Camblong

University of San Francisco, camblong@usfca.edu

Follow this and additional works at: http://repository.usfca.edu/phys

Part of the Physics Commons

\section{Recommended Citation}

Camblong, Horacio E., "Electron Transport in Magnetic Inhomogeneous Media" (1995). Physics and Astronomy. Paper 12.

http://repository.usfca.edu/phys/12

This Article is brought to you for free and open access by the College of Arts and Sciences at USF Scholarship: a digital repository @ Gleeson Library | Geschke Center. It has been accepted for inclusion in Physics and Astronomy by an authorized administrator of USF Scholarship: a digital repository @ Gleeson Library | Geschke Center. For more information, please contact repository@usfca.edu. 


\title{
Electron transport in magnetic inhomogeneous media
}

\author{
Horacio E. Camblong \\ Department of Physics, New York University, New York, New York 10003 \\ and Department of Physics, University of San Francisco, San Francisco, California 94117 \\ Peter M. Levy and Shufeng Zhang \\ Department of Physics, New York University, New York, New York 10003
}

(Received 9 May 1994; revised manuscript received 30 January 1995)

\begin{abstract}
Giant magnetoresistance has been observed in both magnetic multilayers and magnetic granular solids. We develop a framework for unifying these particular realizations of inhomogeneous magnetic media, based on the real-space Kubo formula. It constitutes a spin-dependent form of linear response theory, associated with internal spin-dependent fields arising from spin accumulation; moreover, we discuss the physical meaning of these spin dependences. For magnetic multilayers we discuss the particular cases of collinear and noncollinear configurations, and we consider limiting cases of the elastic mean-free path to inhomogeneity-scale ratio for granular solids. Furthermore, we introduce the concept of magnetically self-averaging systems, which include the current perpendicular to the plane geometry of multilayers and granular solids. In the limit of no spin-flip scattering, we show that there are no length scales associated with the magnetoresistance of self-averaging structures.
\end{abstract}

\section{INTRODUCTION}

The recent interest in the magnetoresistive properties of magnetic multilayered structures and granular films has led to the question of the proper treatment of electron transport in these inhomogeneous media. The main problem is that the sizes of the inhomogeneities (thickness of layers, granular size) are comparable to the average mean free path of the conduction electrons; therefore, transport properties vary from one region to another, and one must find a way of determining the measured transport properties (resistivity or magnetoresistivity) from the local ones. In general, there is no simple equivalent resistor network that is applicable to this situation.

Over the past decade progress has been made in this problem. It was first resolved for multilayers made of dissimilar metals, e.g., $\mathrm{Pd} / \mathrm{Au},{ }^{1}$ and then extended to magnetic multilayers, e.g., Fe-Cr. ${ }^{2}$ On the assumption of homogeneous layers (single grain, single domain), and for current in the plane (CIP) of the layers, the electric field is constant throughout a multilayered structure, so that the problem of finding the measured resistivity and magnetoresistance (MR) is simplified to the point where we have been able to determine the resistivity for arbitrary alignments of the moments of the magnetic layers. ${ }^{3}$

For current perpendicular to the plane (CPP) of the layers in multilayered structures and for granular films the electric field seen by the conduction electrons is not,immediately known-i.e., it is not constant-and we must determine it in order to find the measured resistivity. In general this is quite difficult and this problem has been solved with some simplifying assumptions $;{ }^{4-6}$ principal among them are that we considered only collinear magnetization configurations of the layers or granules, that no spin-flip processes are present, and that the mag- netic layers are single domains. Even with these restrictions we have found it necessary to extend the conventional treatments of electron transport in solids by admitting effective spin-dependent electric fields. ${ }^{4-6}$ Valet and Fert have relaxed the second restriction and considered the effects of spin-flip processes on conduction and magnetoresistance. ${ }^{7}$ Recently, we considered the effects of grains and magnetic domains within the layers, and concluded that they promote spin diffusion even in the absence of spin-flip processes. ${ }^{8}$

In this paper we relax the remaining approximation and consider electron transport in magnetic inhomogeneous media for arbitrary arrangements of magnetic moments. As we show, it is necessary to introduce currents, fields, and conductivity tensors that are off diagonal in the spin space of the conduction electrons. In the next section we derive the constitutive relation between field and current in the linear-response regime. Solutions for the Green's function, self-energy, and bubble conductivity (the simplest one) are outlined in Sec. III. For arbitrary structures the general solutions of the constitutive relations are not known, and we demonstrate the validity of our formalism by considering special cases. In Sec. IV we consider layered structures for CIP and CPP; for single-domain magnetic layers the electric field is constant throughout in the CIP geometry, so that the measured conductivity is written as a double integral over the local conductivities. When we restrict ourselves to non-spin-flip scattering processes, the measured CPP resistivity can be written as a double integral over the local resistivities. To make progress on solutions for general inhomogeneous media, e.g., granular films, we first consider in Sec. V limiting cases of the sizes of the inhomogeneities relative to the electron mean free path. Then in Sec. VI we introduce the concept of magnetic 
self-averaging and discuss the global conductivity (resistivity) and magnetoresistance for those inhomogeneous systems whose transport properties are magnetically selfaveraging. In the concluding Sec. VII we summarize and discuss the trends for the magnetoresistance for arbitrary structures and magnetic configurations.

In the Appendixes we discuss the transformation properties of the spinor matrices that we have introduced in the constitutive relations, we introduce an alternative definition for the internal fields, we derive the equation of continuity for the generalized (off-diagonal) currents, we define path-ordering operators introduced to handle the noncommutativity of the spin matrices appearing in the differential equation for the Green's function and in the expression for the conductivity, and we invert the spinor conductivity in a limiting scenario.

\section{HAMILTONIAN AND LINEAR RESPONSE}

For nonmagnetic (normal) metals the current at a point $\mathbf{r}$ is related to the static electric field at a point $\mathbf{r}^{\prime}$ through the two-point conductivity

$$
\mathbf{j}(\mathbf{r})=\int d^{3} r^{\prime} \boldsymbol{\sigma}\left(\mathbf{r}, \mathbf{r}^{\prime}\right) \cdot \mathbf{E}\left(\mathbf{r}^{\prime}\right),
$$

where $\mathbf{E}\left(\mathbf{r}^{\prime}\right)$ is the internal electric field in the solid and $\boldsymbol{\sigma}\left(\mathbf{r}, \mathbf{r}^{\prime}\right)$ is the microscopic conductivity given by Kubo's linear-response formalism, which in the zero-frequency limit is 9,10

$$
\boldsymbol{\sigma}\left(\mathbf{r}, \mathbf{r}^{\prime}\right)=\lim _{\omega \rightarrow 0} \frac{\Pi\left(\mathbf{r}, \mathbf{r}^{\prime} ; \omega\right)}{\omega}
$$

where $\Pi\left(\mathbf{r}, \mathbf{r}^{\prime} ; \omega\right)$ is the frequency-dependent currentcurrent correlation function,

$$
\Pi\left(\mathbf{r}, \mathbf{r}^{\prime} ; \omega\right)=\int_{0}^{\infty} d \tau e^{i \omega \tau}\left\langle\left[\hat{\mathbf{j}}(\mathbf{r}, t+\tau), \hat{\mathbf{j}}\left(\mathbf{r}^{\prime}, t\right)\right]\right\rangle
$$

and $\hat{\mathbf{j}}(\mathbf{r}, t)$ is the quantum-mechanical current operator. This function is independent of $t$ due to time-translation invariance. The angular brackets denote the expectation value of the commutator taken over all states of the system (we limit ourselves to zero temperature). Therefore, to calculate the conductivity it is necessary to write down the Hamiltonian that describes the conduction electrons.

By making some separation between conduction and localized (core) electrons the one-electron model Hamiltonian for inhomogeneous magnetic structures is

$$
H=H_{0}+V_{\text {scatt }}
$$

with

$$
H_{0}=\frac{p^{2}}{2 m}+V_{\mathrm{pot}, M}(\mathbf{r}, \sigma)
$$

and

$$
V_{\text {scatt }}=\sum_{a}\left(\nu_{a}+j_{a} \hat{\mathbf{M}}_{a} \cdot \hat{\boldsymbol{\sigma}}\right) \delta\left(\mathbf{r}-\mathbf{r}_{a}\right)
$$

where $\hat{\boldsymbol{\sigma}}$ stands for the Pauli spin vector operator and
$\hat{\mathbf{M}}_{a}$ represents the orientation of the magnetization of the corresponding region. We have taken the range of this scattering potential to be zero; this simplifies our treatment of the local scattering without altering the effect we are modeling. The roughness of the interfaces is modeled as a scattering potential in Eq. (2.6); as the scattering is confined to a narrow region, we have represented it as random in the plane of the interface and a $\delta$ function in the third dimension (direction of layer growth). This is the same potential that results from the unitary transformation used by Tešanović et $a{ }^{11}$ to map the rough boundary problem into one with flat surfaces; however, in multilayers it is more correct to think of this scattering plane representing the interfacial region, e.g., the mixed layer of Johnson and Camley, ${ }^{12}$ than resulting from the unitary transformation.

While we have included the Coulomb interaction of the conduction electrons with the background $V_{\text {pot, } M}$ and used it to calculate the wave functions and one-electron propagators, here we do not explicitly consider it between the conduction electrons themselves; this enters the vertex corrections to the conductivity (polarization diagrams). Also we are primarily interested in transport at $T=0 \mathrm{~K}$, so that processes that occur at finite temperature are omitted. Here $V_{\text {pot, } M}$ represents the spindependent potentials of the electrons in the different regions and $M$ denotes the magnetic configuration, e.g., ferromagnetic, antiferromagnetic, or random; for layered structures, its effects on the MR have been considered by several groups. ${ }^{13-17}$ In granular films, this potential is random, and there have been no attempts to include its effect on conduction. As there are sufficient complications arising from the scattering potential, Eq. (2.6), most of the theoretical development to date has been confined to taking $V_{\text {pot }}=$ const (zero)..$^{18}$

For the nonmagnetic regions $j=0$ [see Eq. (2.6)], while at interfaces and in magnetic regions (granules or layers) $j \neq 0$. The majority of the data on the giant MR effect has been on metallic structures with two active ingredients: one magnetic and one nonmagnetic element. If we exclude the substrates, buffer and capping layers, and layers that are used to pin the magnetic layers in spinvalve structures, there are five parameters that characterize the scattering: At the interfaces there is $\nu^{s}$ and $j^{s}$, while for the bulk the parameters are $\nu^{m}, j^{m}$, and $\nu^{\mathrm{nm}}$, where $m \equiv$ magnetic and $\mathrm{nm} \equiv$ nonmagnetic. Alternately we can use $\nu^{s}, \nu^{m}$, and $\nu^{\mathrm{nm}}$ to set the strength of the scattering in the different regions, and $p^{s}$ and $p^{m}$ for the spin dependence, where $p \equiv j / \nu$.

The scattering potential $V_{\text {scatt }}$, Eq. (2.6), refers to the scattering of a single electron by a particular configuration of impurities. While transport properties of very pure systems, e.g., mesoscopic systems, depend on the specific configuration, ${ }^{19} \mathrm{Kohn}$ and Luttinger showed that their mean square deviations from the mean value, normalized by their mean value, tend to zero as the number of impurities tends to infinity. ${ }^{20}$ Therefore, for all but the purest systems in which the transport is "ballistic," and specifically for metallic layered and granular structures in which giant MR has been observed, transport properties are described by functions, such as the self-energy 
and one-particle propagators, which have been averaged over all possible configurations of impurities. This "impurity average" eliminates the dependence of transport properties on the specifics of the impurity configuration.

Electrical transport in metals leads to a redistribution of space charge if the rate at which the electrons are scattered varies from one region to another. For this reason, the field is not the same as the field applied externally; i.e., while one might apply a uniform electric field, the internal field is not uniform in inhomogeneous media. Transport in magnetic metals with spindependent scattering or spin-polarized currents leads to a spatial redistribution of spin as well as charge ${ }^{21}$ this is referred to either as spin accumulation or nonequilibrium (current-driven) magnetization. To account for either spin-dependent scattering or spin-polarized conduction [see Eqs. (2.5) and (2.6)], it is necessary to introduce the spin variables referring to the conduction electrons in the constitutive relation that relates fields to currents. To introduce spin variables we start from the bare external field, not from the internal field defined in Eq. (2.1) for nonmagnetic (normal) metals.

When considering spin-dependent interactions in the electron's Hamiltonian, the electron propagators, currents, and internal fields are spin dependent. If one only considers collinear magnetization configurations (ferromagnetic or antiferromagnetic), it is sufficient to consider the two currents $\mathbf{j}^{\uparrow}$ and $\mathbf{j}^{\downarrow}$ associated with the two spin conduction channels. Even for this simplified scenario, it is necessary to consider spin-dependent fields $\mathbf{E}^{\uparrow}$ and $\mathbf{E}^{\downarrow}$; see Refs. 4-6. In this paper our purpose is twofold: on the one hand, to clarify the meaning of these spindependent fields and, on the other hand, to generalize the theory to arbitrary magnetization configurations and arbitrary choices of the quantization axis.

In order to have a covariant description for arbitrary choices of the quantization axis, we introduce quantities with definite spinor character. This procedure is not just a theoretical nicety; rather, it is dictated by the physical requirement that the values of measurable properties (such as the electrical resistance) be independent of the unphysical (arbitrary) choice of a quantization axis. In Appendix $A$ we discuss the geometrical meaning and properties of such covariant or geometrical quantities. Instead, if one tried to implement a noncovariant or nongeometrical formalism, one would be led into the nonphysical absurdity that the electrical resistance is quantization-axis dependent.

This program can be implemented by defining the generalized spin-dependent current densities

$$
\mathbf{j}_{\alpha \beta}(\mathbf{r})=\left\langle\hat{\mathbf{j}}_{\alpha \beta}(\mathbf{r})\right\rangle
$$

which are expectation values of the spinor current operators $^{22}$

$$
\hat{\mathbf{j}}_{\alpha \beta}(\mathbf{r})=\frac{e \hbar}{m i} \Psi_{\alpha}^{\dagger}(\mathbf{r}) \stackrel{\leftrightarrow}{\nabla}_{\mathbf{r}} \Psi_{\beta}(\mathbf{r})
$$

where

$$
\stackrel{\leftrightarrow}{\nabla}_{\mathbf{r}}=\frac{1}{2}\left(\vec{\nabla}_{\mathbf{r}}-\overleftarrow{\nabla}_{\mathbf{r}}\right)
$$

is the antisymmetric gradient operator, $\Psi_{\alpha}(\mathbf{r})$ is the realspace one-electron field operator, and Greek indices label the two spin channels.

The question arises as to the extent to which the choice of off-diagonal currents is not unique. In effect, for a given choice of a quantization axis, there are two distinct spin channels and (among the currents) only the quantities $\mathbf{j}_{\uparrow}=\mathbf{j}_{++}$and $\mathbf{j}_{\downarrow}=\mathbf{j}_{--}$have a direct physical meaning; for a different set of axes, the corresponding values would be different: $\mathbf{j}_{\uparrow}^{\prime}=\mathbf{j}_{++}^{\prime}$ and $\mathbf{j}_{\downarrow}^{\prime}=\mathbf{j}_{--}^{\prime}$. The requirement that $\mathbf{j}_{\alpha \beta}$ be a second-rank spinor implies that it can be diagonalized for a particular set of axes with physical currents $\mathbf{j}_{\uparrow}^{0}$ and $\mathbf{j}_{\downarrow}^{0}$, and that for any other set of axes both its diagonal and off-diagonal elements be uniquely given in terms of $\mathbf{j}_{\uparrow}^{0}$ and $\mathbf{j}_{\downarrow}^{0}$ and of the corresponding elements of the rotation matrix connecting the two coordinate systems (see Appendix A); therefore, their uniqueness follows.

Once these unique currents have been introduced we can investigate the problem of the internal fields. By introducing the spinor notation above, we find that the spinor current at point 1 is related to the external electric field at point 3 by $^{23}$

$$
\begin{aligned}
\mathbf{j}_{\alpha \beta}(1) & =\int d 3 \sigma_{\alpha \beta}(13) \cdot \mathbf{E}_{\mathrm{ext}}(3) \\
& =-\frac{4}{\pi} \frac{e^{2}}{\hbar}\left(\frac{\hbar^{2}}{2 m}\right)^{2} \int d 2 d 3 d 4\left\{G_{\beta \gamma}(12) \stackrel{\leftrightarrow}{\nabla}_{1} \boldsymbol{\Gamma}_{\gamma \delta}(234) G_{\delta \alpha}(41)\right\} \cdot \mathbf{E}_{\mathrm{ext}}(3)
\end{aligned}
$$

where the Feynman diagram associated with this expression is given in Fig. 1(a). In Eq. (2.10), as well as in all subsequent equations, summation over repeated Greek indices is implied. Here $G(1,2)$ is a one-electron propagator; it is a $2 \times 2$ spin matrix, inasmuch as it satisfies a differential equation which includes a $2 \times 2$ spin scattering matrix which represents the impurity-averaged self-energy; see the next section. The vector vertex function $\Gamma(234)$ is represented by a $2 \times 2$ spin matrix that is given by the integral equation ${ }^{23}$ [see Fig. 1(b)],

$$
\begin{aligned}
\Gamma(234)= & \stackrel{\leftrightarrow}{\nabla}_{3} \mathbb{1} \delta(2,3) \delta(4,3)+V(24) \int d 2^{\prime} d 4^{\prime} G\left(2,2^{\prime}\right) \boldsymbol{\Gamma}\left(2^{\prime} 34^{\prime}\right) G\left(4^{\prime}, 4\right) \\
& +\delta(2,4) \int d 2^{\prime} d 4^{\prime} d 5 V(25) G\left(5,2^{\prime}\right) \boldsymbol{\Gamma}\left(2^{\prime} 34^{\prime}\right) G\left(4^{\prime}, 5\right)
\end{aligned}
$$




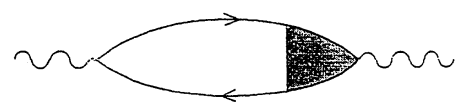

(a)
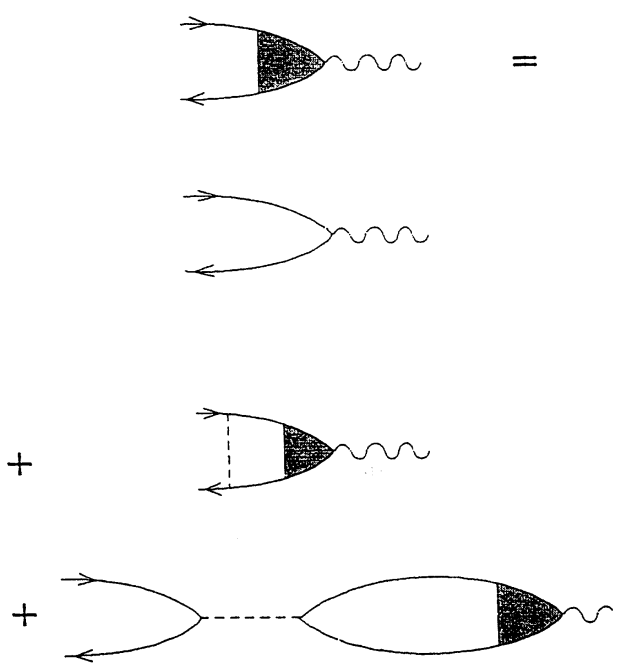

(b)

FIG. 1. (a) Feynman diagram for the conductivity, Eq. (2.10), consisting of electron and hole propagators coupled at the right end to an electric field through a dressed vertex. (b) This shaded portion represents the vertex correction Eq. (2.11); this term consists of three parts: the bare vertex, the corrections due to interactions between electrons, and those coming from the impurity averaging, which couple the electron and hole propagators, and polarization corrections which modify the field seen by the electrons.

where the $V$ 's represent interactions not accounted for in the one-particle propagator $G$, matrix multiplication is implied, $\mathbb{1}$ is a $2 \times 2$ unit matrix, and $\delta(1,2)$ stands for the Dirac delta function $\delta\left(\mathbf{r}_{1}-\mathbf{r}_{2}\right)$. The first term is the bare vertex, the second one represents vertex corrections to the electron-hole propagators (two-particle Green's functions), which represent correlations due to scattering at different sites that are lost when one uses Green's functions with impurity-averaged self-energies, and the last term accounts for polarization effects (Coulomb interactions) in the medium that contribute to the total internal field as distinguished from the external field $\mathbf{E}_{\text {ext }}(3) .{ }^{24}$

Once the Green's functions $G$ are known, the integral equation for the vertex function can be solved (in principle at least); however, this is rarely done. What is important about Eq. (2.11) is that it allows one to check that one has made conserving approximations. ${ }^{25}$ Some of the relations that define these approximations are referred to as Ward identities. ${ }^{9,10}$

While the first term in Eq. (2.11) represents the interaction of an electron with the external field, the other terms represent the effects of the other electrons that have rearranged themselves in response to the field. By using the vertex function, we define the effective local field acting on an electron in response to an external field as follows. First, we define a scalar vertex function ${ }^{9}$

$$
\Gamma_{\gamma \delta}(234)=\stackrel{\leftrightarrow}{\nabla}_{2} \Gamma_{\gamma \delta}(234)
$$

where the gradient operates on the Green's functions when it is placed in Eq. (2.10). Then, we write

$$
\int d 3 \Gamma_{\gamma \delta}(234) \mathbf{E}_{\mathrm{ext}}(3)=\mathbf{E}_{\gamma \delta}(2) \delta(2,4),
$$

where we have confined the corrections in the second term of the vertex function, Eq. (2.11), to those that can be represented by a local field, i.e., where 2 and 4 are one and the same position in space. For example, to lowest order in the density of scatterers, vertex corrections due to impurity averaging are local ${ }^{9,10}$ (the applicability of this to interfaces requires further study). Upon placing Eq. (2.13) in Eq. (2.10) we find

$$
\begin{aligned}
\mathbf{j}_{\alpha \beta}(1)= & -\frac{4}{\pi} \frac{e^{2}}{\hbar}\left(\frac{\hbar^{2}}{2 m}\right)^{2} \int d 2\left\{G_{\beta \gamma}(12) \stackrel{\leftrightarrow}{\nabla}_{1} \stackrel{\leftrightarrow}{\nabla}_{2}\right. \\
& \left.\times G_{\delta \alpha}(21)\right\} \cdot \mathbf{E}_{\gamma \delta}(2) .
\end{aligned}
$$

This can be put in the form of Eq. (2.1), but now with spin indices,

$$
\mathbf{j}_{\alpha \beta}(\mathbf{r})=\int d^{3} r^{\prime} \boldsymbol{\sigma}_{\alpha \beta, \gamma \delta}\left(\mathbf{r}, \mathbf{r}^{\prime}\right) \cdot \mathbf{E}_{\gamma \delta}\left(\mathbf{r}^{\prime}\right)
$$

where $\sigma_{\alpha \beta, \gamma \delta}\left(\mathbf{r}, \mathbf{r}^{\prime}\right)$ is a two-point fourth-rank spinor conductivity. It represents the terms in the curly brackets in Eq. (2.14) which contain only the contribution from the bubble diagram in the Kubo formula; this is the diagram in Fig. 1(a) without the vertex corrections, Fig. 1(b), i.e., with just a bare vertex. This spinor conductivity is proportional to the bubble part of the current-current correlation of the spinor currents, i.e., $\sigma_{\alpha \beta, \gamma \delta}\left(\mathbf{r}, \mathbf{r}^{\prime}\right) \propto$ $\left\langle\left[\hat{\mathbf{j}}_{\alpha \beta}(\mathbf{r}), \hat{\mathbf{j}}_{\gamma \delta}\left(\mathbf{r}^{\prime}\right)\right]\right\rangle$; its transformation properties are discussed in Appendix A. Equations (2.10)-(2.14) are to be interpreted as Matsubara Green's functions; they lead to combinations of retarded and advanced Green's functions, so that the two-point spinor conductivity is explicitly written $\mathrm{as}^{26}$

$$
\begin{aligned}
& \boldsymbol{\sigma}_{\alpha \beta, \gamma \delta}\left(\mathbf{r}, \mathbf{r}^{\prime}\right) \\
& =\frac{4}{\pi} \frac{e^{2}}{\hbar}\left(\frac{\hbar^{2}}{2 m}\right)^{2} A_{\beta \gamma}\left(\mathbf{r}, \mathbf{r}^{\prime}\right) \stackrel{\leftrightarrow}{\nabla}_{\mathbf{r}} \stackrel{\leftrightarrow}{\nabla}_{\mathbf{r}^{\prime}} A_{\delta \alpha}\left(\mathbf{r}^{\prime}, \mathbf{r}\right),
\end{aligned}
$$

where

$$
\begin{aligned}
A_{\alpha \beta}\left(\mathbf{r}, \mathbf{r}^{\prime}\right) & =\frac{i}{2}\left[G_{\alpha \beta}^{\mathrm{ret}}\left(\mathbf{r}, \mathbf{r}^{\prime}\right)-G_{\alpha \beta}^{\mathrm{adv}}\left(\mathbf{r}, \mathbf{r}^{\prime}\right)\right] \\
& =\frac{i}{2}\left\{G_{\alpha \beta}^{\mathrm{ret}}\left(\mathbf{r}, \mathbf{r}^{\prime}\right)-\left[G_{\beta \alpha}^{\mathrm{ret}}\left(\mathbf{r}^{\prime}, \mathbf{r}\right)\right]^{*}\right\}
\end{aligned}
$$

is the density of states function and where the property

$$
\left[G_{\alpha \beta}^{\mathrm{ret}}\left(\mathbf{r}, \mathbf{r}^{\prime}\right)\right]^{*}=G_{\beta \alpha}^{\mathrm{adv}}\left(\mathbf{r}^{\prime}, \mathbf{r}\right)
$$


has been explicitly used.

From Eqs. (2.13) and (2.15) it is clear that the charge and spin accumulations attendant to conduction in inhomogeneous magnetic structures are accounted for in the effective internal field $\mathbf{E}_{\gamma \delta}\left(\mathbf{r}^{\prime}\right)$, Eq. (2.13), which has absorbed the vertex corrections [the third term in Eq. (2.11)]. It would be incorrect to include such effects when calculating the conductivity $\boldsymbol{\sigma}_{\alpha \boldsymbol{\beta}, \gamma \delta}\left(\mathbf{r}, \mathbf{r}^{\prime}\right)$ if one uses the effective fields defined in Eq. $(2.13) ;^{27}$ it would be tantamount to including polarization effects to evaluate the current-current correlation function when one is relating the current to the total electric field. ${ }^{24}$ In fact, by starting with the vertex equation, Eq. (2.11), we have shown in Ref. 31 that the vertex function $\Gamma_{\gamma \delta}(232)$ [note $4=2$ from Eq. (2.13)], at large distances between points 3 and 2 , satisfies the diffusion equation that has been used in quasiclassical treatments of transport in inhomogeneous magnetic structures to account for spin and charge accumulation. ${ }^{7,28-31}$

Finding the global or measurable conductivity, i.e., the net current density across the boundaries of a sample for an applied potential, for inhomogeneous structures from the local conductivity, Eq. (2.16), is by no means straightforward. The main difficulty arises from the lack of symmetry in general, which leads to complicated distributions (magnitudes and directions) for both current densities and internal fields. If one could evaluate the internal field $\mathbf{E}_{\gamma \delta}\left(\mathbf{r}^{\prime}\right)$ from the vertex function, Eq. (2.13), one could find the current density from Eq. (2.14); this is equivalent to solving the conductivity in Eq. (2.10) when the field $\mathbf{E}$ is simply related to the applied potential. Provided one has taken care to use currentconserving approximations for the vertex function, one is assured that the steady-state current density, i.e., the trace over spin space of the spinor current density, i.e., $\mathbf{j}(\mathbf{r})=\operatorname{Tr}[\mathbf{j}(\mathbf{r})]=\sum_{\alpha} \mathbf{j}_{\alpha}(\mathbf{r})$, is conserved. ${ }^{25}$ Thus,

$$
\boldsymbol{\nabla} \cdot \mathbf{j}(\mathbf{r})=0
$$

because $\partial \rho(\mathbf{r}) / \partial t=0$ in the steady state. Similarly, in the steady state, the spinor current densities satisfy the equation (see Appendix C)

$$
\boldsymbol{\nabla} \cdot \mathbf{j}_{\alpha \beta}(\mathbf{r})=w_{\alpha \beta}(\mathbf{r}) .
$$

The right-hand side of Eq. (2.20) looks like charge buildup or loss, but it is due to spin-flip processes; thus, we define spin-flip processes as those that give rise to finite $w_{\alpha \beta}(\mathbf{r})$; conversely, when $w_{\alpha \beta}(\mathbf{r})=0$, we say these are no spin-flip processes. It will be important to distinguish those situations with $w_{\alpha \beta}(\mathbf{r}) \neq 0$, which reduce the giant MR effect due to spin mixing, ${ }^{7,32}$ from those in the absence of spin flips but where the off-diagonal elements of the spinor current density $\mathbf{j}_{\alpha \beta}(\mathbf{r})$ are due to one's using quantization axes at an angle to the direction of the magnetization in a region [see Eq. (2.6)]. In this latter case, as we show in Appendix $\mathrm{C}, w_{\alpha \beta}(\mathbf{r})=0$, and the steady-state current density is conserved for each spin component

$$
\boldsymbol{\nabla} \cdot \mathbf{j}_{\boldsymbol{\alpha} \boldsymbol{\beta}}(\mathbf{r})=0 .
$$

Kane et $a l .{ }^{33}$ have shown directly from the Kubo for- mula that $\boldsymbol{\nabla} \cdot \boldsymbol{\sigma}\left(\mathbf{r}, \mathbf{r}^{\prime}\right)=0$ and $\boldsymbol{\sigma}\left(\mathbf{r}, \mathbf{r}^{\prime}\right) \cdot \overleftarrow{\nabla^{\prime}}=0$ (with the gradient operator acting to the left), so that the equation of continuity is trivially satisfied, independently of the electric field distribution $\mathbf{E}\left(\mathbf{r}^{\prime}\right)$ in Eq. (2.1). Similarly we show in Appendix $\mathrm{C}$ that the spinor currents $\mathbf{j}_{\alpha \beta}(\mathbf{r})$ are divergenceless when there are no spin-flip processes. However, the bubble conductivity (2.16) and its equivalent, when taking the spin trace of Eq. (2.16), are not divergenceless. Therefore, imposing the constraint of Eq. (2.20) or of Eq. (2.21) on the current density given by Eq. (2.15) provides a set of equations that allows one in some cases to determine the effective internal fields $\mathbf{E}_{\gamma \delta}\left(\mathbf{r}^{\prime}\right)$, namely,

$$
\int d^{3} r^{\prime} \nabla_{\mathbf{r}} \cdot \sigma_{\alpha \beta, \gamma \delta}\left(\mathbf{r}, \mathbf{r}^{\prime}\right) \cdot \mathbf{E}_{\gamma \delta}\left(\mathbf{r}^{\prime}\right)=w_{\alpha \beta}(\mathbf{r}) .
$$

Another condition comes from the definitions of the internal field, Eq. (2.13), and the vertex function, Eq. (2.11); then

$$
\int_{\mathcal{C}} \mathbf{E}_{\gamma \delta}\left(\mathbf{r}^{\prime}\right) \cdot d \mathbf{r}^{\prime}=V \delta_{\gamma \delta}
$$

where $V$ is the voltage applied to the outer boundaries of the structure and the line integral is evaluated along the current path $\mathcal{C}$ from one boundary to the other. In effect, the condition

$$
\int_{\mathcal{C}} \mathbf{E}_{\alpha \alpha}\left(\mathbf{r}^{\prime}\right) \cdot d \mathbf{r}^{\prime}=V
$$

has to be satisfied for both channels $\alpha$ and for an arbitrary choice of the quantization axis; then, Eq. (2.23) follows, as the only spinor with coordinate-independent and equal diagonal elements is a multiple of the identity, which amounts to a scalar.

In cases where there is some symmetry (layered structures), we will show that one can solve for the fields and currents. For more complicated geometries (granular films), we resort to an ansatz based on some degree of randomness to find the measured resistivity and MR.

\section{IIY. EVALUATION OF "BUBBLE CONDUCTIVITY"}

In Sec. II we derived the two-point spinor conductivity, Eq. (2.16); it is given in terms of one-particle propagators $G_{\alpha \beta}\left(\mathbf{r}, \mathbf{r}^{\prime}\right)$. As defined by Eq. (2.16), they are impurity-averaged Green's functions; parenthetically, if one used the Green's function for one specific impurity configuration, there would be no vertex corrections entering Eq. (2.11) from impurity averaging; this would simplify the expression (2.13) for the internal field, albeit at the expense of a more complicated Green's function $G_{\alpha \beta}$.

To calculate the conductivity, Eq. (2.16), it is necessary to obtain the impurity-averaged one-electron propagators or Green's functions $\langle G(\varepsilon)\rangle_{\mathcal{I}}$ (these brackets denote impurity average with respect to an ensemble $\mathcal{I}$ of impurities). This function can be expressed in terms of the self-energy $\Sigma(\epsilon)$ through Dyson's equation

$$
\langle G(\varepsilon)\rangle=\left[\varepsilon-H_{0}-\Sigma(\varepsilon)\right]^{-1} .
$$


There are two different quantum approaches to calculating the one-electron propagators and conductivity for our model Hamiltonian, Eq. (2.4). One is based on the local self-energy, which is applicable to the dilute and weak-scattering limit of impurities; a real-space representation is used to find, without further approximation or limits, the propagators and the two-point conductivity. In this approach one has concluded that the quasiclassical and the real-space quantum treatments (based on a local self-energy) produce the same magnetotransport properties, provided the effect of quantum interference and quantum size effects can be neglected. ${ }^{30,34,35}$ The second approach uses a nonlocal self-energy built up from the one-site $t$ matrix; here, one attempts to take into account distant but strong scattering from interfaces. By using this approach, approximate solutions have been found for the one-point conductivity, Eq. (4.7), for multilayered structures by using the Kubo formalism in momentum space. ${ }^{3,36}$ In some limiting cases both approaches give identical results; otherwise, they provide different free parameters whenever the local scattering rates vary significantly from region to region and the mean free paths are of the order of the inhomogeneity length scales. However, at the present time there is not enough experimental evidence in favor of either one or the other approach. It should be pointed out that the reciprocal-space approach has two limitations: (i) It is based on a decoupling procedure (introduced in Ref. 3) that constitutes an uncontrolled approximation whose validity has not been firmly established; (ii) in its present form it does not yield a two-point conductivity, a restriction that does not allow it to be generalized from multilayers to magnetic inhomogeneous media.

Here we present a real-space quantum approach to transport in inhomogeneous three-dimensional structures. ${ }^{26}$ This approach has been proposed in Refs. 5, 34, and 37 for quasi-one-dimensional layered structures. Our treatment is an extension of the formulation of Refs. 5 and 34 for multilayered structures. In this treatment we take $H_{0}$ in Eq. (2.4) to be that of free electrons and we neglect the differences in the potentials between regions; i.e., we set $V_{\text {pot }}=$ const (zero).

To describe transport properties, one focuses on the imaginary part of the self-energy; the real part will be subsumed in $H_{0}$ [see Eq. (3.1)]. For weak scattering and the dilute limit of the impurity concentration in the Hamiltonian (2.4) the imaginary part of the local self-energy is simply determined by the local scattering rates $^{30,34}$

$$
\begin{aligned}
\Delta(\mathbf{r}) \equiv & -\operatorname{Im}[\Sigma(\mathbf{r})] \\
= & \pi n_{\mathrm{imp}}(\mathbf{r}) \rho\left(\varepsilon_{F}\right)\left[\nu^{2}(\mathbf{r})+j^{2}(\mathbf{r})\right. \\
& +2 \nu(\mathbf{r}) j(\mathbf{r}) \hat{\mathbf{M}}(\mathbf{r}) \cdot \hat{\boldsymbol{\sigma}}],
\end{aligned}
$$

where $\rho\left(\varepsilon_{F}\right)$ is the density of states per unit volume at the Fermi level and $\hat{\mathbf{M}}(\mathbf{r})$ is a unit vector in the direction of the local magnetization. By defining

$$
\mathcal{G}\left(\mathbf{r}, \mathbf{r}^{\prime}\right)=\left(\hbar^{2} / 2 m\right) G\left(\mathbf{r}, \mathbf{r}^{\prime}\right),
$$

we find Eq. (3.1) is written as

$$
\left\{\nabla_{\mathbf{r}}^{2}+\left[k_{F}^{2}+i 2 m \Delta(\mathbf{r}) / \hbar^{2}\right]\right\} \mathcal{G}\left(\mathbf{r}, \mathbf{r}^{\prime}\right)=\delta\left(\mathbf{r}-\mathbf{r}^{\prime}\right) ;
$$

that is, it has the "Helmholtz form"

$$
\left[\nabla_{\mathbf{r}}^{2}+k^{2}(\mathbf{r})\right] \mathcal{G}\left(\mathbf{r}, \mathbf{r}^{\prime}\right)=\delta\left(\mathbf{r}-\mathbf{r}^{\prime}\right)
$$

with a complex wave number

$$
k(\mathbf{r})=\left[k_{F}^{2}+i 2 m \Delta(\mathbf{r}) / \hbar^{2}\right]^{1 / 2},
$$

where we have suppressed the unit $2 \times 2$ matrix to make the equation more transparent. One solves this equation by a straightforward WKB integration ${ }^{30,34}$ (see Appendix D)

$$
\mathcal{G}\left(\mathbf{r}, \mathbf{r}^{\prime}\right)=-\frac{1}{4 \pi\left|\mathbf{r}-\mathbf{r}^{\prime}\right|} P_{\mathbf{r}^{\prime} \rightarrow \mathbf{r}} \exp \left[i \int_{\Gamma\left[\mathbf{r}, \mathbf{r}^{\prime}\right]} d s^{\prime \prime} k\left(\mathbf{r}^{\prime \prime}\right)\right],
$$

where the integral extends along the oriented straight path $\Gamma\left[\mathbf{r}, \mathbf{r}^{\prime}\right]$ that starts at point $\mathbf{r}^{\prime}$ and ends up at point $\mathbf{r}$, and $P_{\mathbf{r}^{\prime} \rightarrow \mathbf{r}}$ is the path-ordering operator that reorders the noncommuting $2 \times 2$ scattering matrices in the exponential series from the point $\mathbf{r}^{\prime}$ to the point $\mathbf{r}$ and from right to left; the properties of the path-ordering operator are discussed in Appendix D. In effect, Eq. (3.7), which is a straightforward generalization of the solution $-e^{i k R} /(4 \pi R)$ (with $R=\left|\mathbf{r}-\mathbf{r}^{\prime}\right|$ ) of the constant- $k$ GreenHelmholtz equation, is an exact solution of the Green's function (3.5), for locally homogeneous media, as can be verified by direct substitution (see Appendix D for a detailed derivation of this result).

In the quasiclassical regime, the electron propagates throughout the solid satisfying the condition

$$
k_{F} \gg \xi(\mathbf{r})
$$

where

$$
\xi(\mathbf{r})=\left(\frac{2 m}{\hbar^{2} k_{F}}\right) \Delta(\mathbf{r})
$$

which is of course equivalent to

$$
\varepsilon_{F} \gg \Delta(\mathbf{r}) \text {. }
$$

This amounts to a complex wave number $k(\mathbf{r})$ with a "large" real part given by the Fermi wave number and a "small" imaginary part given by the scattering matrix, i.e.,

$$
k(\mathbf{r}) \approx k_{F}+\frac{i}{2} \xi(\mathbf{r}) .
$$

Substituting Eq. (3.11) in Eq. (3.7),

$$
\begin{aligned}
\mathcal{G}\left(\mathbf{r}, \mathbf{r}^{\prime}\right) \approx- & \frac{1}{4 \pi\left|\mathbf{r}-\mathbf{r}^{\prime}\right|} \exp \left[i k_{F}\left|\mathbf{r}-\mathbf{r}^{\prime}\right|\right] \\
& \times P_{\mathbf{r}^{\prime} \rightarrow \mathbf{r}} \exp \left[-\frac{1}{2} \xi\left(\mathbf{r}, \mathbf{r}^{\prime}\right)\left|\mathbf{r}-\mathbf{r}^{\prime}\right|\right]
\end{aligned}
$$

where

$$
\xi\left(\mathbf{r}, \mathbf{r}^{\prime}\right)=\frac{1}{\left|\mathbf{r}-\mathbf{r}^{\prime}\right|} \int_{\Gamma\left[\mathbf{r}, \mathbf{r}^{\prime}\right]} d s^{\prime \prime} \xi\left(\mathbf{r}^{\prime \prime}\right)
$$


Notice that both $\xi(\mathbf{r})$ and $\xi\left(\mathbf{r}, \mathbf{r}^{\prime}\right)$ have dimensions of inverse length, and can be interpreted as inverse local and "two-point" mean free paths; in particular,

$$
l(\mathbf{r})=[\xi(\mathbf{r})]^{-1}
$$

is the local mean free path probed by electrons as they propagate through a "local" region. In Eqs. (3.7) and (3.12), the path-ordering operator $P_{\mathbf{r}^{\prime} \rightarrow \mathbf{r}}$ is required due to the noncommutativity of spin matrices that is characteristic of noncollinear magnetization configurations and the corresponding Green's function is not symmetric under the exchange of the points $\mathbf{r}$ and $\mathbf{r}^{\prime}$; in effect, in the presence of spin-dependent scattering, Eq. (2.6), the oneparticle propagator $G\left(\mathbf{r}, \mathbf{r}^{\prime}\right)$, and the self-energy $\Sigma(\mathbf{r})$, as well as $\Delta(\mathbf{r})$ and $\xi\left(\mathbf{r}, \mathbf{r}^{\prime}\right)$ are $2 \times 2$ spin matrices that cannot be simultaneously diagonalized except for the partic- ular case of collinear magnetization configurations.

The physical interpretation of Eqs. (3.7) and (3.12) is as follows: They describe an electron propagating with the "large" complex wave number $k(\mathbf{r})$, which yields a wave of real spatial frequency $k_{F}$ with an exponential decay with characteristic decay lengths given by the elements of the inverse scattering matrix $l(\mathbf{r})$ [Eq. (3.14)]. In this quasiclassical or "geometrical" picture, the electron singles out quasiclassical trajectories, i.e., straight lines $\Gamma\left[\mathbf{r}, \mathbf{r}^{\prime}\right]$ connecting the two given points.

Equation (3.12) represents the retarded Green's function, which corresponds to outgoing waves, $e^{i k_{F} R}$. Instead, the corresponding advanced Green's function, which corresponds to incoming waves, $e^{-i k_{F} R}$, can be calculated by means of the symmetry relation (2.18), and used to compute the density of states, Eq. (2.17) which reads

$$
\begin{aligned}
\mathcal{A}_{\boldsymbol{\alpha} \beta}\left(\mathbf{r}, \mathbf{r}^{\prime}\right) & =\frac{\hbar^{2}}{2 m} A_{\alpha \beta}\left(\mathbf{r}, \mathbf{r}^{\prime}\right) \\
& =\frac{i}{2}\left\{\mathcal{G}_{\alpha \beta}^{\mathrm{ret}}\left(\mathbf{r}, \mathbf{r}^{\prime}\right)-\left[\mathcal{G}_{\beta \alpha}^{\mathrm{ret}}\left(\mathbf{r}^{\prime}, \mathbf{r}\right)\right]^{*}\right\} \\
& =\left(-\frac{i}{4 \pi\left|\mathbf{r}-\mathbf{r}^{\prime}\right|}\right) \frac{1}{2}\left\{\mathcal{P}_{\alpha \beta}\left(\mathbf{r}, \mathbf{r}^{\prime}\right) e^{i k_{F} R}-\left[\mathcal{P}_{\beta \alpha}\left(\mathbf{r}^{\prime}, \mathbf{r}\right)\right]^{*} e^{-i k_{F} R}\right\} \\
& =\frac{1}{4 \pi\left|\mathbf{r}-\mathbf{r}^{\prime}\right|}\left[\sin k_{F} R\right] \mathcal{P}_{\alpha \beta}\left(\mathbf{r}, \mathbf{r}^{\prime}\right)
\end{aligned}
$$

where we have defined the "path-ordered transport exponent"

$$
\mathcal{P}\left(\mathbf{r}, \mathbf{r}^{\prime}\right)=\left\{P_{\mathbf{r}^{\prime} \rightarrow \mathbf{r}} \exp \left[-\frac{1}{2} \xi\left(\mathbf{r}, \mathbf{r}^{\prime}\right)\left|\mathbf{r}-\mathbf{r}^{\prime}\right|\right]\right\}
$$

an object that satisfies the symmetry property

$$
\left[\mathcal{P}_{\alpha \beta}\left(\mathbf{r}, \mathbf{r}^{\prime}\right)\right]^{*}=\mathcal{P}_{\beta \alpha}\left(\mathbf{r}^{\prime}, \mathbf{r}\right) \text {. }
$$

Property (3.17) expresses the fact that if the ordering of scattering matrices is reversed by an exchange of the points $\mathbf{r}$ and $\mathbf{r}^{\prime}$, all products are transposed, and the Hermiticity of the scattering matrices does the rest [see Appendix A for the symmetry properties of physical quantities and Appendix D, for the properties of the pathordering operator and for a proof of Eq. (3.17)].

In order to compute the two-point conductivity from the Kubo formula, Eq. (2.16), we need to differentiate Eq. (3.15). In doing so, we again apply the quasiclassical approximation. In this context, it means that length scales $R \lesssim k_{F}^{-1}$ are not probed; thus, the limit $k_{F} R \gg 1$ can be systematically applied. Calling $\mathbf{R}=\mathbf{r}-\mathbf{r}^{\prime}$, we find that

$$
\begin{aligned}
\boldsymbol{\nabla}_{\mathbf{r}} \mathcal{A}_{\alpha \beta}\left(\mathbf{r}, \mathbf{r}^{\prime}\right)= & \frac{1}{4 \pi R} \mathcal{P}_{\alpha \beta}\left(\mathbf{r}, \mathbf{r}^{\prime}\right)\left[\nabla_{\mathbf{r}} \sin k_{F} R\right] \\
& \times\left[1+O\left(\frac{1}{k_{F} R}\right)\right] .
\end{aligned}
$$

Thus, using $\nabla_{\mathbf{R}}=\nabla_{\mathbf{r}}=-\nabla_{\mathbf{r}^{\prime}}$, we get

$$
\begin{aligned}
\mathcal{A}_{\boldsymbol{\beta}}\left(\mathbf{r}, \mathbf{r}^{\prime}\right) & \stackrel{\leftrightarrow}{\nabla}_{\mathbf{r}} \stackrel{\leftrightarrow}{\nabla}_{\mathbf{r}^{\prime}} \mathcal{A}_{\delta \alpha}\left(\mathbf{r}^{\prime}, \mathbf{r}\right) \\
= & \left(\frac{1}{4 \pi R}\right)^{2} \mathcal{P}_{\beta \gamma}\left(\mathbf{r}, \mathbf{r}^{\prime}\right) \mathcal{P}_{\delta \alpha}\left(\mathbf{r}^{\prime}, \mathbf{r}\right) \\
& \times\left(\sin k_{F} R\right) \stackrel{\leftrightarrow}{\nabla}_{\mathbf{r}} \stackrel{\leftrightarrow}{\nabla}_{\mathbf{r}^{\prime}}\left(\sin k_{F} R\right)\left[1+O\left(\frac{1}{k_{F} R}\right)\right] .
\end{aligned}
$$

Therefore, asymptotically in the limit of the large parameter $k_{F} R$, only the leading term in the equations above is relevant. This leading term can be computed by using

$$
\stackrel{\leftrightarrow}{\nabla}_{\mathbf{r}} \stackrel{\leftrightarrow}{\nabla}_{\mathbf{r}^{\prime}}=-\stackrel{\leftrightarrow}{\nabla}_{\mathbf{R}} \stackrel{\leftrightarrow}{\nabla}_{\mathbf{R}}=-\left[\overleftarrow{\nabla}_{\mathbf{R}}^{2}+\vec{\nabla}_{\mathbf{R}}^{2}-2 \overleftarrow{\nabla}_{\mathbf{R}} \vec{\nabla}_{\mathbf{R}}\right] / 4
$$

and $\boldsymbol{\nabla}_{\mathbf{R}} f(R)=\mathbf{n} d f / d R$, where $\mathbf{n}=\mathbf{R} / R$ is a unit vector from $\mathbf{r}$ to $\mathbf{r}^{\prime}$, whence

$$
\left(\sin k_{F} R\right) \stackrel{\leftrightarrow}{\nabla}_{\mathbf{r}} \stackrel{\leftrightarrow}{\nabla}_{\mathbf{r}^{\prime}}\left(\sin k_{F} R\right)=\frac{1}{2} k_{F}^{2} \mathbf{n n} .
$$

It follows that in the quasiclassical limit, the Kubo formula, Eq. (2.16), yields

$$
\begin{aligned}
\boldsymbol{\sigma}_{\alpha \beta, \gamma \delta}\left(\mathbf{r}, \mathbf{r}^{\prime}\right) \\
=\frac{3 C_{D}}{4 \pi} \frac{\mathbf{n} \mathbf{n}}{\left|\mathbf{r}-\mathbf{r}^{\prime}\right|^{2}}\left\{P_{\mathbf{r}^{\prime} \rightarrow \mathbf{r}} \exp \left[-\frac{1}{2} \xi\left(\mathbf{r}, \mathbf{r}^{\prime}\right)\left|\mathbf{r}-\mathbf{r}^{\prime}\right|\right]\right\}_{\beta \gamma} \\
\quad \times\left\{P_{\mathbf{r} \rightarrow \mathbf{r}^{\prime}} \exp \left[-\frac{1}{2} \xi\left(\mathbf{r}, \mathbf{r}^{\prime}\right)\left|\mathbf{r}-\mathbf{r}^{\prime}\right|\right]\right\}_{\delta \alpha}
\end{aligned}
$$


where

$$
C_{D}=\frac{e^{2} k_{F}^{2}}{6 \pi^{2} \hbar}=\frac{n e^{2}}{2 m v_{F}}
$$

(with $n$ being the total density of conduction electrons) is a constant with dimensions of conductivity/length.

\section{LAYERED STRUCTURES}

In cases where there are spatial or spin symmetries, or in certain limits of the characteristic length scales $d_{\text {in }}$ which describe the inhomogeneity of the structure, the general constitutive relation between field and current, given by Eqs. (2.15) and (3.21), simplifies and it is possible to evaluate explicitly the global conductivity (resistivity) and MR. Here we consider the simplifications due to geometrical and magnetic symmetries.

We designate the layers to lie in the $x-y$ plane and the growth direction to be the $z$ axis. We do not focus on the atomic potentials or on granularity (multidomains) of the layers, and as random impurity averages are taken over the $x-y$ planes of the layers, ${ }^{30}$ the planes are homo- geneous. The conductivity, Eq. (2.10) or Eq. (2.16), can be written as

$$
\boldsymbol{\sigma}\left(\mathbf{r}, \mathbf{r}^{\prime}\right)=\boldsymbol{\sigma}\left(\boldsymbol{\rho}-\boldsymbol{\rho}^{\prime} ; z, z^{\prime}\right)
$$

where $\rho=(x, y)$ and we have temporarily suppressed the spin indices. By taking the Fourier transform of Eq. (2.15) with respect to $\left(\boldsymbol{\rho}-\boldsymbol{\rho}^{\prime}\right)$, we find it is written as

$$
\mathbf{j}_{\alpha \beta}(\mathbf{k}, z)=\int d z^{\prime} \boldsymbol{\sigma}_{\alpha \beta, \gamma \delta}\left(\mathbf{k} ; z, z^{\prime}\right) \cdot \mathbf{E}_{\gamma \delta}\left(\mathbf{k}, z^{\prime}\right) .
$$

We are interested in cases where the fields are uniform over the layers and we assume the magnetic domains in the layers are large compared to the mean free paths. Thus, we take $\mathbf{k}=0$ and suppress this index, so that Eq. (4.2) is written as

$$
\mathbf{j}_{\alpha \beta}(z)=\int d z^{\prime} \boldsymbol{\sigma}_{\alpha \beta, \gamma \delta}\left(z, z^{\prime}\right) \cdot \mathbf{E}_{\gamma \delta}\left(z^{\prime}\right)
$$

and we are left with a one-dimensional (1D) problem for the spatial dependence of currents and fields. by ${ }^{5,34}$

$$
\begin{aligned}
& \boldsymbol{\sigma}_{\alpha \beta, \gamma \delta}\left(z, z^{\prime}\right)=\frac{3 C_{D}}{2} \int_{1}^{\infty} d t \\
& {\left[\frac{1}{2}\left(\frac{1}{t}-\frac{1}{t^{3}}\right) \mathbb{1}_{\|}+\frac{1}{t^{3}} \mathbf{e}_{z} \mathbf{e}_{z}\right]\left\{P_{z^{\prime} \rightarrow z} \exp \left[-\frac{t}{2} \int_{z_{<}}^{z>} d z^{\prime \prime} \xi\left(z^{\prime \prime}\right)\right]\right\}_{\beta \gamma} } \\
& \times\left\{P_{z \rightarrow z^{\prime}} \exp \left[-\frac{t}{2} \int_{z_{<}}^{z_{>}} d z^{\prime \prime} \xi\left(z^{\prime \prime}\right)\right]\right\}_{\delta \alpha}
\end{aligned}
$$

where $C_{D}$ is defined by Eq. (3.22), the substitution $t=$ $\left|\mathbf{r}-\mathbf{r}^{\prime}\right| /\left|z-z^{\prime}\right|$ has been made, $\xi(z)=\left(2 m / \hbar^{2} k_{F}\right) \Delta(z)$, $\mathbb{1}_{\|}$is the unit tensor in the plane of the layers, $\mathbf{e}_{z}$ is the unit vector in the $z$ direction, and integration with respect to the in-plane azimuthal angle has rendered the tensor diagonal.

For fields parallel to the plane of the layers (CIP), the electric field $\mathbf{E}_{\gamma \delta}(z)$ is a constant. From the condition (2.23) we find,

$$
E_{\gamma \delta}^{\mathrm{CIP}}=\frac{V}{L_{T}} \delta_{\gamma \delta} \equiv E \delta_{\gamma \delta}
$$

when $L_{T}$ is the length of the sample in the in-plane direction, i.e., the square root of the cross sectional area of the multilayered structure. Therefore, the current density for CIP,

$$
j_{\alpha \beta}(z)=\sigma_{\alpha \beta}^{(\|)}(z) E,
$$

is proportional to the one-point conductivity

$$
\sigma_{\alpha \beta}^{(\|)}(z)=\sum_{\gamma} \int d z^{\prime} \sigma_{\alpha \beta, \gamma \gamma}^{(\|)}\left(z, z^{\prime}\right),
$$

where $\sigma^{(\|)}$denotes the in-plane component of the con- ductivity tensor. The measured current per unit area, or average current density $\overline{j(z)}=I / A$, where $j(z)=$ $\operatorname{Tr}[j(z)]=\sum_{\alpha} j_{\alpha \alpha}(z)$, is

$$
\langle j(z)\rangle=\frac{1}{L} \int d z j(z) \equiv \sigma_{\mathrm{CIP}} E
$$

where $\sigma_{\text {CIP }}$ the global or measured CIP conductivity,

$$
\begin{aligned}
\sigma_{\mathrm{CIP}} & =\frac{1}{L} \iint d z d z^{\prime} \sum_{\alpha, \gamma} \sigma_{\alpha \alpha, \gamma \gamma}^{(\|)}\left(z, z^{\prime}\right) \\
& =\frac{1}{L} \iint d z d z^{\prime} \operatorname{Tr}\left[\sigma^{(\|)}\left(z, z^{\prime}\right)\right]
\end{aligned}
$$

and $L$ is the length of the sample in the $z$ direction. In Eq. (4.9), Tr is a shorthand for the double partial trace explicitly shown. The remarkable feature of these results for CIP is that one can determine the resistivity and MR, for this particular geometry, because the field distribution is trivial. The result, Eqs. (4.8) and (4.9), reinforces the point of Dieny et al. ${ }^{38}$ that it is more relevant to talk about conductivities than resistivities for CIP.

For currents perpendicular to the plane of the layers (CPP), the internal field $E_{\gamma \delta}(z)$ is not constant. However, from the continuity equation, Eq. (2.21), one gains 
information on the current density. Therefore, it is better to express the electric field in terms of the current, i.e., to invert the CPP component of Eq. (4.3), i.e.,

$$
E_{\alpha \beta}(z)=\int d z^{\prime} \rho_{\alpha \beta, \gamma \delta}^{(\perp)}\left(z, z^{\prime}\right) j_{\gamma \delta}\left(z^{\prime}\right)
$$

where $\rho^{(\perp)}$ is the perpendicular component of the resistivity tensor. The Kubo formula yields the conductivity; in order to find the two-point resistivity, one must solve the integral equation

$$
\int d z^{\prime \prime} \sigma_{\alpha \beta, \gamma \delta}^{(\perp)}\left(z, z^{\prime \prime}\right) \rho_{\gamma \delta, \alpha^{\prime} \beta^{\prime}}^{(\perp)}\left(z^{\prime \prime}, z^{\prime}\right)=\delta\left(z-z^{\prime}\right) \delta_{\alpha \alpha^{\prime}} \delta_{\beta \beta^{\prime}},
$$

that is, invert the matrix

$$
\begin{aligned}
\rho_{\alpha \beta, \gamma \delta}^{(\perp)}\left(z, z^{\prime}\right) & =\left[\sigma_{\alpha \beta, \gamma \delta}^{(\perp)}\right]^{-1}\left(z, z^{\prime}\right) \\
& \neq\left[\sigma_{\alpha \beta, \gamma \delta}^{(\perp)}\left(z, z^{\prime}\right)\right]^{-1}
\end{aligned}
$$

which is unwieldy; see Sec. V A and Appendix E. In limiting cases this amounts to inverting a one-point conductivity $\sigma_{\alpha \beta, \gamma \delta}^{(\perp)}(z)(4 \times 4$ spin matrix $)$; if there is sufficient spin symmetry present so that no spin indices are necessary, the one-point resistivity $\rho(z)$ is just $1 / \sigma(z)$.

The voltage drop per unit length of the sample or average electric field $\overline{E_{\alpha \beta}}$ is, from Eqs. (2.23) and (4.10),

$$
\begin{aligned}
\overline{E_{\alpha \beta}} & =\frac{1}{L} \int d z E_{\alpha \beta}(z) \\
& =\frac{1}{L} \iint d z d z^{\prime} \rho_{\alpha \beta, \gamma \delta}^{(\perp)}\left(z, z^{\prime}\right) j_{\gamma \delta}\left(z^{\prime}\right) \\
& =\bar{E} \delta_{\alpha \beta} .
\end{aligned}
$$

The current density $j_{\gamma \delta}\left(z^{\prime}\right)$ for the CPP geometry satisfies the ordinary differential equation [see Eq. (2.20)]

$$
\partial_{z} j_{\alpha \beta}(z)=w_{\alpha \beta}(z) .
$$

One trivial solution is for $w_{\alpha \beta}(z)=0$, i.e., for the case of no spin flips or no spin mixing of the two spin channels. In this case, $j_{\alpha \beta}(z)$ is a constant, and Eq. (4.13) reduces to

$$
\bar{E} \delta_{\alpha \beta}=\rho_{\alpha \beta, \gamma \delta}^{\mathrm{CPP}} j_{\gamma \delta}
$$

where

$$
\rho_{\alpha \beta, \gamma \delta}^{\mathrm{CPP}}=\frac{1}{L} \iint d z d z^{\prime} \rho_{\alpha \beta, \gamma \delta}^{(\perp)}\left(z, z^{\prime}\right) .
$$

This expression can be inverted, i.e.,

$$
j_{\alpha \beta}=\left(\left[\rho^{\mathrm{CPP}}\right]^{-1}\right)_{\alpha \beta, \gamma \delta} \delta_{\gamma \delta} \bar{E}=\left(\left[\rho^{\mathrm{CPP}}\right]^{-1}\right)_{\alpha \beta, \gamma \gamma} \bar{E},
$$

where $\left(\left[\rho^{\mathrm{CPP}}\right]^{-1}\right)_{\alpha \beta, \gamma \delta}$ are the elements of the inverse of the average two-point resistivity; this is not the same as the average two-point conductivity. The total current is

$$
\begin{aligned}
j=\operatorname{Tr}[j] & =\sum_{\alpha} j_{\alpha \alpha} \\
& =\bar{E} \sum_{\alpha \gamma}\left(\left[\rho^{\mathrm{CPP}}\right]^{-1}\right)_{\alpha \alpha, \gamma \gamma},
\end{aligned}
$$

where the square brackets indicate one is taking the inverse of a spin matrix; see Appendix E. Thus, the conductivity for CPP when $w_{\alpha \beta}(z)=0$ [see Eq. (4.14)] is

$\sigma_{\mathrm{CPP}}(w=0)=\sum_{\alpha \gamma}\left(\left[\rho^{\mathrm{CPP}}\right]^{-1}\right)_{\alpha \alpha, \gamma \gamma}=\operatorname{Tr}\left(\left[\rho^{\mathrm{CPP}}\right]^{-1}\right)$,

where Tr stands for the double partial trace.

By comparing Eqs. (4.9) and (4.19) one notes that the two conductivities are quite different. The CIP global conductivity is a complete sum of the two-point conductivities over the entire sample and over all spin indices; in some limiting cases it is analogous to conduction for a set of resistors in parallel. The CPP conductivity, Eq. (4.19)is a sum over the conductivities for each spin channel $\alpha$; the conductivity in each channel comes from taking the inverse of the resistivity $\rho^{\mathrm{CPP}}$, Eq. (4.16), which is arrived at by summing over the two-point resistivities. It is analogous to a set of resistors in series, for a specific spin index. ${ }^{39}$

\section{GLOBAL CONDUCTIVITY FOR INHOMOGENEOUS SYSTEMS}

\section{A. Introduction}

Finding the global or measurable conductivity for inhomogeneous structures from the two-point conductivity is by no means straightforward. The problem has a twofold complexity: its spin structure and its nonlocal structure (the former arising from spin dependence and the related covariance of physical quantities and the latter from the very existence of inhomogeneities).

This complexity can be clearly seen from the generalization of Eqs. (4.10)-(4.12) to three-dimensional inhomogeneous structures. In effect, inversion of the linear constitutive relationship, Eq. (2.15), allows one to express the internal fields $\mathbf{E}_{\boldsymbol{\alpha} \beta}(\mathbf{r})$, which in general are not constant, in terms of the current [because, from the continuity equation, Eq. (2.21), one makes a statement about the current density]; therefore,

$$
\mathbf{E}_{\alpha \beta}(\mathbf{r})=\int d^{3} r^{\prime} \boldsymbol{\rho}_{\alpha \beta, \gamma \delta}\left(\mathbf{r}, \mathbf{r}^{\prime}\right) \cdot \mathbf{j}_{\gamma \delta}\left(\mathbf{r}^{\prime}\right)
$$

where $\boldsymbol{\rho}_{\alpha \beta, \gamma \delta}\left(\mathbf{r}, \mathbf{r}^{\prime}\right)$ is the resistivity tensor, which is the solution of the $3 \mathrm{D}$ generalization of integral equation (4.11), namely,

$$
\begin{aligned}
& \int d^{3} r^{\prime \prime} \boldsymbol{\sigma}_{\alpha \beta, \gamma \delta}\left(\mathbf{r}, \mathbf{r}^{\prime \prime}\right) \cdot \boldsymbol{\rho}_{\gamma \delta, \alpha^{\prime} \beta^{\prime}}\left(\mathbf{r}^{\prime \prime}, \mathbf{r}^{\prime}\right) \\
&=\mathbb{1}_{3} \delta\left(\mathbf{r}-\mathbf{r}^{\prime}\right) \delta_{\alpha \alpha^{\prime}} \delta_{\beta \beta^{\prime}}
\end{aligned}
$$


where $\mathbb{1}_{3}$ is the unit tensor in three-dimensional space.

The spin-index complexity, as we will see, can be trivially reduced in the so-called homogeneous limit (Sec. V B). Otherwise, it is convenient to use the following matrix representation, based on "bi-indices." Let $s \equiv(\alpha \beta)$ and $r \equiv(\gamma \delta)$; i.e., $s$ and $r$ may take any of the following four values: $s, r=(++),(--),(+-),(-+)$ (which we will always arrange in that order); then, Eq. (2.15) acquires the matrix form

$$
\mathbf{j}_{s}(\mathbf{r})=\int d^{3} r^{\prime} \boldsymbol{\sigma}_{s r}\left(\mathbf{r}, \mathbf{r}^{\prime}\right) \cdot \mathbf{E}_{r}\left(\mathbf{r}^{\prime}\right)
$$

where the currents $\mathbf{j}_{s}(\mathbf{r})$ and fields $\mathbf{E}_{s}(\mathbf{r})$ become $4 \times$ 1 "column" matrices and the two-point conductivity $\sigma_{s r}\left(\mathbf{r}, \mathbf{r}^{\prime}\right)$ becomes a $4 \times 4$ matrix (with respect to biindices). The details of this procedure, particularly regarding transformation properties when different quantization axes are chosen, are discussed in Appendixes A and E; from a practical viewpoint, it suffices to view Eq. (5.3) as a matrix equation, for every choice of axes. In principle, this allows one to carry out an inversion of Eqs. (2.15) and (5.3), by using Eqs. (5.1) and (5.2), i.e.,

$$
\mathbf{E}_{s}(\mathbf{r})=\int d^{3} r^{\prime} \boldsymbol{\rho}_{s r}\left(\mathbf{r}, \mathbf{r}^{\prime}\right) \cdot \mathbf{j}_{r}\left(\mathbf{r}^{\prime}\right)
$$

thus, the two-point resistivity tensor is the $4 \times 4$ inverse matrix with respect to spin bi-indices of the two-point conductivity tensor,

$$
\begin{aligned}
\boldsymbol{\rho}_{s r}\left(\mathbf{r}, \mathbf{r}^{\prime}\right) & =\left[\boldsymbol{\sigma}_{s r}\right]^{-1}\left(\mathbf{r}, \mathbf{r}^{\prime}\right) \\
& \neq\left[\boldsymbol{\sigma}_{s r}\left(\mathbf{r}, \mathbf{r}^{\prime}\right)\right]^{-1} .
\end{aligned}
$$

This inversion can be explicitly carried out in both the homogeneous limit (Sec. VB) and in the local limit (Sec. V C), but it is most useful for situations that resemble CPP, which are discussed in Sec. VIA.

On the other hand, the complexity associated with spatial inhomogeneities is also trivially reduced in the homogeneous limit in terms of the average of the scattering; due to symmetries, this complexity is also reduced in the case of multilayers. For granular solids it can be handled only in an approximate way; the main difficulty arises from the lack of symmetry, which leads to complicated distributions for both current densities and internal fields.

\section{B. Homogeneous limit}

As a first step towards determining the global conductivity for inhomogeneous structures, we consider the homogeneous limit, which is defined as the limit when all mean free paths are much larger than all inhomogeneity length scales.

In the homogeneous limit, for both multilayers and granular solids, the two-point function $\xi\left(\mathbf{r}, \mathbf{r}^{\prime}\right)$ [Eq. (3.13)] has a unique limit, independent of $\mathbf{r}$ and $\mathbf{r}^{\prime}$ when $R=$ $\left|\mathbf{r}-\mathbf{r}^{\prime}\right| \rightarrow \infty$, with the possible exception of a subfamily of paths $\Gamma\left[\mathbf{r}, \mathbf{r}^{\prime}\right]$ in the plane of the layers for multilayers; in other words, the average of the scattering $\Delta(\mathbf{r})$ is well defined for almost all paths. In actuality, in defining spatial averages, $R$ has an upper bound set up by the size $L$ of the sample; therefore, what is characteristic of selfaveraging systems is the existence of a minimum length scale $D_{\text {sa }}$, such that for $D_{\text {sa }} \lesssim R \ll L$, the function $\xi\left(\mathbf{r}, \mathbf{r}^{\prime}\right)$ becomes asymptotically a constant. For magnetic metallic systems, the function $\xi\left(\mathbf{r}, \mathbf{r}^{\prime}\right)$ is a spin matrix; its self-averaging limit is of the form $\bar{\xi}=\bar{\xi}_{0}+\bar{\xi} \cdot \hat{\boldsymbol{\sigma}}$. As a consequence of this definition, for any region $\mathcal{R}$ of dimensions of the order of $D_{\text {sa }}$ (or greater), the average of the scattering over a volume $V$,

$$
\bar{\Delta}=\frac{1}{V[\mathcal{R}]} \int_{\mathcal{R}} d^{3} r \Delta(\mathbf{r})=\bar{\Delta}_{0}+\hat{\boldsymbol{\sigma}} \cdot \overline{\boldsymbol{\Delta}},
$$

is independent of the chosen region $\mathcal{R}$ for both multilayers and granular solids. In Eq. (5.6), we have defined

$$
\bar{\Delta}_{0}=\pi \rho\left(\varepsilon_{F}\right) \int \frac{d^{3} r}{V} n(\mathbf{r})\left[v^{2}(\mathbf{r})+j^{2}(\mathbf{r})\right]
$$

and

$$
\overline{\boldsymbol{\Delta}}=2 \pi \rho\left(\varepsilon_{F}\right) \int \frac{d^{3} r}{V} n(\mathbf{r}) v(\mathbf{r}) j(\mathbf{r}) \hat{\mathbf{M}}(\mathbf{r}) .
$$

As a consequence, when all the local mean free paths are much larger than all inhomogeneity length scales (homogeneous limit), the two-point conductivity, Eq. (3.21), becomes effectively a function of only $R=\left|\mathbf{r}-\mathbf{r}^{\prime}\right|$, namely [from Eq. (3.21)],

$$
\begin{aligned}
\boldsymbol{\sigma}_{\alpha \beta, \gamma \delta}\left(\mathbf{r}, \mathbf{r}^{\prime}\right)= & \frac{3 C_{D}}{4 \pi} \frac{\mathbf{n} \mathbf{n}}{R^{2}}\left[P_{\mathbf{r}^{\prime} \rightarrow \mathbf{r}} \exp \left(-\frac{1}{2} \bar{\xi} R\right)\right]_{\beta \gamma} \\
& \times\left[P_{\mathbf{r} \rightarrow \mathbf{r}^{\prime}} \exp \left(-\frac{1}{2} \bar{\xi} R\right)\right]_{\delta \alpha},
\end{aligned}
$$

for $R \gtrsim D_{\text {sa }}$, where $D_{\text {sa }}$ refers to the self-averaging length. Choosing the particular "privileged" axis that diagonalizes $\bar{\xi}$, path ordering becomes superfluous, i.e.,

$$
\left[P_{\mathbf{r}^{\prime} \rightarrow \mathbf{r}} \exp \left(-\frac{1}{2} \bar{\xi} R\right)\right]_{\alpha \beta}=\delta_{\alpha \beta}\left[\exp \left(-\frac{1}{2} \bar{\xi} R\right)\right]_{\alpha \alpha},
$$

whence

$$
\begin{aligned}
\boldsymbol{\sigma}_{\alpha \beta, \gamma \delta}\left(\mathbf{r}, \mathbf{r}^{\prime}\right)= & \frac{3 C_{D}}{4 \pi} \frac{\mathbf{n} \mathbf{n}}{R^{2}}\left[\exp \left(-\frac{1}{2} \bar{\xi} R\right)\right]_{\beta \beta} \\
& \times\left[\exp \left(-\frac{1}{2} \bar{\xi} R\right)\right]_{\alpha \alpha} \delta_{\beta \gamma} \delta_{\delta \alpha} .
\end{aligned}
$$

In Eqs. (5.10) and (5.11) no summation is implied. In particular, using

$$
\delta_{\beta \gamma} \delta_{\gamma \alpha}=\delta_{\alpha \beta}
$$

the following reduction can be carried out: 


$$
\begin{aligned}
\sum_{\gamma} \boldsymbol{\sigma}_{\alpha \beta, \gamma \gamma}\left(\mathbf{r}, \mathbf{r}^{\prime}\right) & \equiv \boldsymbol{\sigma}_{\alpha \beta, \gamma \delta}\left(\mathbf{r}, \mathbf{r}^{\prime}\right) \delta_{\delta \gamma} \\
& =\frac{3 C_{D}}{4 \pi} \frac{\mathbf{n} \mathbf{n}}{R^{2}}\left[\exp \left(-\frac{1}{2} \bar{\xi} R\right)\right]_{\beta \beta}\left[\exp \left(-\frac{1}{2} \bar{\xi} R\right)\right]_{\alpha \alpha} \delta_{\beta \gamma} \delta_{\delta \alpha} \delta_{\delta \gamma} \\
& =\frac{3 C_{D}}{4 \pi} \frac{\mathbf{n} \mathbf{n}}{R^{2}}\left[\exp \left(-\frac{1}{2} \bar{\xi} R\right)\right]_{\beta \beta}\left[\exp \left(-\frac{1}{2} \bar{\xi} R\right)\right]_{\alpha \alpha} \delta_{\alpha \beta} \\
& =\delta_{\alpha \beta} \boldsymbol{\sigma}_{\alpha}^{\text {homog }}(R)
\end{aligned}
$$

where

$$
\boldsymbol{\sigma}_{\alpha}^{\text {homog }}=\frac{3 C_{D}}{4 \pi} \frac{\mathbf{n} \mathbf{n}}{R^{2}}[\exp (-\bar{\xi} R)]_{\alpha \alpha}
$$

[in Eqs. (5.13) and (5.14), indices $\alpha$ and $\beta$ are not summed over].

On the other hand, considering that the internal fields vary over distances of the order of the inhomogeneity length scales (which are much smaller than the effective mean free paths), the relationship between fields and current densities, Eq. (2.15), is effectively reduced to

$$
\mathbf{j}_{\alpha \beta}(\mathbf{r})=\left[\int d^{3} r^{\prime} \boldsymbol{\sigma}_{\alpha \beta, \gamma \delta}\left(\mathbf{r}, \mathbf{r}^{\prime}\right)\right] \cdot \overline{\mathbf{E}_{\gamma \delta}}
$$

where $\overline{\mathbf{E}_{\gamma \delta}}$ stands for the average of the field in the medium (and which can be understood simply as the average over the mean free path), which, in accordance with Eq. (2.23), is a scalar,

$$
\overline{\mathbf{E}_{\gamma \delta}}=\delta_{\gamma \delta} \overline{\mathbf{E}}
$$

where $\overline{\mathbf{E}}=V / L$ (with $L$ being the length of the sample) is just the average external electric field over the sample. Substituting Eq. (5.16) in Eq. (5.15), and using Eq. (5.13),

$$
\begin{aligned}
\mathbf{j}_{\alpha \beta}(\mathbf{r}) & =\left\{\int d^{3} r^{\prime}\left[\boldsymbol{\sigma}_{\alpha \beta, \gamma \delta}\left(\mathbf{r}, \mathbf{r}^{\prime}\right) \delta_{\gamma \delta}\right]\right\} \cdot \overline{\mathbf{E}} \\
& =\delta_{\alpha \beta}\left[\int d^{3} r^{\prime} \boldsymbol{\sigma}_{\alpha}^{\text {homog }}(R)\right] \cdot \overline{\mathbf{E}}
\end{aligned}
$$

Thus, the current densities become effectively diagonal in the homogeneous limit, with diagonal elements

$$
\mathbf{j}_{\alpha}=\left[\int d^{3} r^{\prime} \boldsymbol{\sigma}_{\alpha}^{\text {homog }}(R)\right] \cdot \overline{\mathbf{E}},
$$

and they are also uniform as a result of the two-point conductivity being a function of $R$ only; in effect, the integral in Eq. (5.17) can be evaluated by a straightforward exponential integration,

$$
\int d^{3} r^{\prime} \boldsymbol{\sigma}_{\alpha}^{\text {homog }}(R)=C_{D} \bar{\xi}_{\alpha}^{-1}=\bar{\rho}_{\alpha}^{-1}
$$

yielding the simple constitutive relationship

$$
\mathbf{j}_{\alpha \beta}=\delta_{\alpha \beta}\left(\bar{\rho}_{\alpha}\right)^{-1} \overline{\mathbf{E}}
$$

or

$$
\overline{\mathbf{E}}=\overline{\rho_{\alpha}} \mathbf{j}_{\alpha},
$$

provided that the axis diagonalizing $\bar{\Delta}$ is chosen. If we now revert to an arbitrary quantization axis, and recall that $\bar{\xi}_{\alpha}$ are the eigenvalues of $\bar{\xi}_{\alpha \beta}$, we obtain

$$
\mathbf{j}_{\alpha \beta}=C_{D} \bar{\xi}_{\alpha \beta}^{-1} \overline{\mathbf{E}}
$$

The global conductance can be calculated from

$$
\begin{aligned}
\mathbf{j}=\sum_{\alpha} \mathbf{j}_{\alpha \alpha} & =C_{D} \sum_{\alpha} \bar{\xi}_{\alpha \alpha}^{-1} \overline{\mathbf{E}} \\
& =C_{D} \operatorname{Tr}\left(\bar{\xi}^{-1}\right) \overline{\mathbf{E}}
\end{aligned}
$$

whence the global conductivity $\sigma$ becomes

$$
\sigma=\rho^{-1}=C_{D} \operatorname{Tr}\left(\bar{\xi}^{-1}\right) ;
$$

i.e., the two-current model is satisfied,

$$
\rho^{-1}=\sum_{\alpha} \rho_{\alpha}^{-1}
$$

with

$$
\begin{aligned}
\rho_{\alpha} & =\left[C_{D}\left(\bar{\xi}^{-1}\right)_{\alpha \alpha}\right]^{-1} \\
& =C_{D}^{-1}\left(\frac{2 m}{\hbar^{2} k_{F}}\right)\left(\left[\bar{\Delta}^{-1}\right]_{\alpha \alpha}\right)^{-1} \\
& =\frac{12 \pi^{2} m}{e^{2} k_{F}^{3} \hbar}\left(\left[\bar{\Delta}^{-1}\right]_{\alpha \alpha}\right)^{-1} .
\end{aligned}
$$

Therefore, from Eq. (5.26), we conclude that the electrical resistance for each spin channel is completely determined by the average scattering $\bar{\Delta}$ in the medium. Notice that this equation reduces to the two-independentcurrent model and is therefore valid provided the spin diffusion length is much larger than both the elastic mean free paths and the inhomogeneity length scales; under this condition the steady-state current density is constrained to satisfy the continuity equation for each spin component, Eq. (2.21).

\section{Local limit}

The opposite limiting case, the local limit, corresponds to the regime when all local mean free paths are much smaller than the inhomogeneity length scales, in which case the linear response of Eq. (2.15) becomes local. The 
precise statement of this locality property follows from considering that

$$
\exp \left[\frac{-\xi\left(\mathbf{r}, \mathbf{r}^{\prime}\right) R}{2}\right] \approx \exp \left[-\frac{1}{2} \xi(\mathbf{r}) R\right],
$$

for $R \gg[\xi(\mathbf{r})]^{-1}$. Equation (5.27) is peaked about $R=$ 0 and leads to a $\delta\left(\mathbf{r}-\mathbf{r}^{\prime}\right)$, after appropriate handling of the spin indices, a procedure that is carried out in Appendix E. As a result, we can define a $4 \times 4$ local conductivity matrix $\sigma_{s r}(\mathbf{r})$, such that

$$
\lim _{R \gg \xi(\mathbf{r})^{-1}} \boldsymbol{\sigma}_{s r}\left(\mathbf{r}, \mathbf{r}^{\prime}\right)=\delta\left(\mathbf{r}-\mathbf{r}^{\prime}\right) \boldsymbol{\sigma}_{s r}\left(\mathbf{r}^{\prime}\right) .
$$

Then,

$$
\left[\sigma_{s r}(\mathbf{r})\right]=C_{D}\left\{\left[\xi_{0}(\mathbf{r})\right]^{2}-[\boldsymbol{\xi}(\mathbf{r})]^{2}\right\}^{-1}\left(\begin{array}{c}
\xi_{--}-\frac{1}{2} \frac{\left|\xi_{-+}\right|^{2}}{\xi_{0}} \\
\frac{1}{2} \frac{\left|\xi_{-+}\right|^{2}}{\xi_{0}} \\
-\frac{1}{2} \xi_{--} \frac{\xi_{-+}}{\xi_{0}} \\
-\frac{1}{2} \xi_{--} \frac{\xi_{-+}^{*}}{\xi_{0}}
\end{array}\right.
$$

$$
\begin{aligned}
\mathbf{j}_{s}(\mathbf{r}) & =\int d^{3} r^{\prime} \delta\left(\mathbf{r}-\mathbf{r}^{\prime}\right) \boldsymbol{\sigma}_{s r}\left(\mathbf{r}^{\prime}\right) \cdot \mathbf{E}_{r}\left(\mathbf{r}^{\prime}\right) \\
& =\boldsymbol{\sigma}_{s r}(\mathbf{r}) \cdot \mathbf{E}_{r}(\mathbf{r})
\end{aligned}
$$

thus the inverse of the local $4 \times 4$ conductivity matrix, that is, the local resistivity matrix $\rho_{s r}(\mathbf{r})$, satisfies

$$
\mathbf{E}_{s}(\mathbf{r})=\boldsymbol{\rho}_{s r}(\mathbf{r}) \cdot \mathbf{j}_{r}(\mathbf{r}) .
$$

A lengthy computation (see Appendix E) yields the following two-point conductivity matrix in the local limit:

$$
\sigma_{s r}(\mathbf{r})=\mathbb{1}_{3} \sigma_{s r}(\mathbf{r})
$$

(where $\mathbb{1}_{3}$ is the unit tensor in three dimensions), with elements where the star stands for complex conjugate and where $\xi_{++}(\mathbf{r}), \xi_{--}(\mathbf{r}), \xi_{-+}(\mathbf{r})$, and $\xi_{+-}(\mathbf{r})$ are the elements of the local reciprocal mean free path matrix,

$$
[\xi(\mathbf{r})]=\xi_{0}(\mathbf{r}) \mathbb{1}+\hat{\boldsymbol{\sigma}} \cdot \boldsymbol{\xi}(\mathbf{r})=\left(\begin{array}{ll}
\xi_{++}(\mathbf{r}) & \xi_{+-}(\mathbf{r}) \\
\xi_{-+}(\mathbf{r}) & \xi_{--}(\mathbf{r})
\end{array}\right)
$$

(where $\mathbb{1}$ is the $2 \times 2$ unit matrix), which is proportional to the local scattering matrix $\Delta(\mathbf{r})$. Explicitly,

$$
\begin{aligned}
& \xi_{ \pm \pm}(\mathbf{r})=\xi_{0}(\mathbf{r}) \pm \xi_{z}(\mathbf{r})=\xi_{0}(\mathbf{r}) \pm|\boldsymbol{\xi}(\mathbf{r})| \cos \theta, \\
& \xi_{ \pm \mp}(\mathbf{r})=\xi_{x}(\mathbf{r}) \mp i \xi_{y}(\mathbf{r})=|\boldsymbol{\xi}(\mathbf{r})| \sin \theta e^{\mp i \varphi} .
\end{aligned}
$$

In the equations above $\theta$ and $\varphi$ are the polar angles of the "vector" $\boldsymbol{\xi}$ with respect to the quantization axis. Moreover, in Eq. (5.32), for the sake of notational simplicity, we have omitted explicit reference to the $\mathbf{r}$ dependence of the reciprocal mean free path $\xi(\mathbf{r})$.

Inversion of the $4 \times 4$ matrix of Eq. (5.32) yields the $4 \times 4$ resistivity matrix (see Appendix E)

$$
\boldsymbol{\rho}_{s r}(\mathbf{r})=\mathbb{1}_{3} \rho_{s r}(\mathbf{r})
$$

with

$$
\left[\rho_{s r}(\mathbf{r})\right]=C_{D}^{-1}\left(\begin{array}{cccc}
\xi_{++} & 0 & \frac{1}{2} \xi_{+-} & \frac{1}{2} \xi_{-+} \\
0 & \xi_{--} & \frac{1}{2} \xi_{+-} & \frac{1}{2} \xi_{-+} \\
\frac{1}{2} \xi_{+-} & \frac{1}{2} \xi_{+-} & 0 & \xi_{0} \\
\frac{1}{2} \xi_{-+} & \frac{1}{2} \xi_{-+} & \xi_{0} & 0
\end{array}\right),
$$

where we have again omitted explicit reference to the $\mathbf{r}$ dependence of the reciprocal mean free path $\xi(\mathbf{r})$.

Using Eqs. (5.30) and (5.36) is by no means trivial, even after performing all the necessary calculations to obtain the local resistivity. The main difficulty comes when there exists an arbitrary distribution of inhomogeneities in the medium; we will consider a particular randomness property in Sec. VI A to evaluate from it the global conductance for granular solids.

\section{Multilayers}

For the particular case of multilayers, which are characterized by in-plane translational invariance, the twopoint conductivity with its arguments $z$ and $z^{\prime}$ can be easily obtained by integrating the in-plane coordinates $\boldsymbol{\rho}-\boldsymbol{\rho}^{\prime}$ of the conductivity; see Eq. (4.4). For CIP the internal electric field induced by an external uniform field is uniform, due to the in-plane translational invariance of the multilayers. The global conductivity can be found by integrating the two-point conductivity of Eq. (4.4) twice, with respect to both arguments $z$ and $z^{\prime}$ [see Eq. (4.9)]; the resulting CIP conductivity exhibits a characteristic exponential dependence with respect to the thicknesses of the different layers, and the magnetoresistance vanishes exponentially in the local limit.

The solution for the CPP conductivity is obtained by rewriting the current density $\mathbf{j}(\mathbf{r})$, from Eqs. (2.15) and (3.21), as an angular average and a radial average, weighted with the conductivity kernel. A possible solution for the internal field, from Eqs. (2.15) and (3.21), is that $\mathbf{E}_{\gamma \delta}\left(\mathbf{r}^{\prime}\right)$ be proportional to the local scattering rate $\Delta_{\gamma \delta}\left(\mathbf{r}^{\prime}\right)$, as follows from the formula 


$$
\begin{aligned}
& \int_{0}^{\infty} d R\left(\left\{P_{\mathbf{r}^{\prime} \rightarrow \mathbf{r}} \exp \left[-\frac{R}{2} \xi\left(\mathbf{r}, \mathbf{r}^{\prime}\right)\right]\right\}_{\beta \gamma} \xi_{\gamma \lambda}\left(\mathbf{r}^{\prime}\right)\left\{P_{\mathbf{r} \rightarrow \mathbf{r}^{\prime}} \exp \left[-\frac{R}{2} \xi\left(\mathbf{r}, \mathbf{r}^{\prime}\right)\right]\right\}_{\delta \alpha}\right. \\
& \left.+\left\{P_{\mathbf{r}^{\prime} \rightarrow \mathbf{r}} \exp \left[-\frac{R}{2} \xi\left(\mathbf{r}, \mathbf{r}^{\prime}\right)\right]\right\}_{\beta \lambda} \xi_{\delta \gamma}\left(\mathbf{r}^{\prime}\right)\left\{P_{\mathbf{r} \rightarrow \mathbf{r}^{\prime}} \exp \left[-\frac{R}{2} \xi\left(\mathbf{r}, \mathbf{r}^{\prime}\right)\right]\right\}_{\gamma \alpha}\right) \\
& =\int d\left(\left\{P_{\mathbf{r}^{\prime} \rightarrow \mathbf{r}} \exp \left[-\frac{R}{2} \xi\left(\mathbf{r}, \mathbf{r}^{\prime}\right)\right]\right\}_{\beta \lambda}\left\{P_{\mathbf{r} \rightarrow \mathbf{r}^{\prime}} \exp \left[-\frac{R}{2} \xi\left(\mathbf{r}, \mathbf{r}^{\prime}\right)\right]\right\}_{\delta \alpha}\right)=\delta_{\beta \lambda} \delta_{\delta \alpha}
\end{aligned}
$$

where $R=\left|\mathbf{r}-\mathbf{r}^{\prime}\right|$ [see Eqs. (3.13) and (3.21)]. This "local" solution can be implemented only when the current density $\mathbf{j}_{\alpha \beta}$ is a constant, which is precisely the case for CPP: $\mathbf{E}(\mathbf{r})=C_{D}^{-1}[\xi(\mathbf{r}) \mathbf{j}+\mathbf{j} \xi(\mathbf{r})] / 2$, where the fields, currents, and strength of scattering are matrices, and a matrix product is implied; as the currents, fields, and scattering matrices can be all diagonalized simultaneously in a local region (reduction to physical diagonal form), one can write simply $\mathbf{E}(\mathbf{r})=C_{D}^{-1} \xi(\mathbf{r}) \mathbf{j}$, or explicitly $\mathbf{E}_{\alpha \beta}(\mathbf{r})=C_{D}^{-1} \xi_{\alpha \gamma}(\mathbf{r}) \mathbf{j}_{\gamma \beta}$. These expressions agree with the particular limiting results of Eqs. (5.20), (5.26), and (5.36). Thus, for the CPP geometry, the corresponding global resistivity is proportional to the average scattering for each spin channel; thus, the CPP geometry for multilayers exhibits a self-averaging behavior (all transport properties are determined by the average scattering) not only in the homogeneous limit, but for all length scales, and the magnetoresistance is scale independent and does not vanish in the local limit. ${ }^{40}$ This result can be described with the aid of the following current-line picture: Current lines sample all the scattering in the medium; later this picture will be generalized to granular solids, for which we will see that there are some restrictions.

The case of multilayers illustrates that there are two radically different behaviors for the magnetoresistance: (a) when it vanishes exponentially with respect to the average distance between magnetic regions (like for the CIP geometry of multilayers); (b) when it does not vanish exponentially and is independent of average distance between magnetic regions (like for the CPP geometry of multilayers). We will refer to the latter category as magnetically self-averaging systems.

\section{MAGNETICALLY SELF-AVERAGING SYSTEMS}

\section{A. Granular solids}

For magnetic precipitates in nonmagnetic matrices, there is no symmetry, other than a certain degree of randomness in the positions and shapes of the granules (precipitates). Here one is left with the general threedimensional (spatial) problem where the field distribution is unspecified and the only constraint on the current density is given by either Eq. (2.20) or Eq. (2.21). One can intuit that conduction in granular solids is similar to the CPP case, as follows. The one condition on transport in granular films is on the current density. Therefore, as indicated in Sec. V A, it is preferable to express the electric field in terms of the current (for the reason outlined in Sec. IV) and to invert Eq. (2.15), i.e., Eq. (5.1). By integrating both sides of Eq. (5.1) along a current path $\mathcal{C}$ we find, by using Eq. (2.23),

$$
V \delta_{\alpha \beta}=\int d^{3} r^{\prime}\left[\int_{\mathcal{C}} d \mathbf{r} \cdot \boldsymbol{\rho}_{\alpha \beta, \gamma \delta}\left(\mathbf{r}, \mathbf{r}^{\prime}\right)\right] \cdot \mathbf{j}_{\gamma \delta}\left(\mathbf{r}^{\prime}\right)
$$

If one now considers, due to the randomness of this structure, that the current density and resistivity function in square brackets at a point $\mathbf{r}^{\prime}$ are not correlated, one averages over them separately and arrives at a result analogous to that for CPP in multilayered structures [see Eqs. (4.15) and (4.16)]. This current-line picture suggests that the global resistivity is proportional to the average scattering $\bar{\Delta}_{\alpha}$ sampled in each spin channel by the current lines, like for the CPP case. This hypothesis provides an upper limit to the resistivity; the actual resistivity is less because the current takes the path of least resistance. Therefore there is some correlation between the current density and resistivity function in Eq. (6.1).

Let us see how the randomness condition above implies that the global resistance is completely determined by $\bar{\Delta}_{\alpha}$, at least in the local limit (and, of course, in the homogeneous limit). In effect, the local resistivity matrix, Eq. (5.36), can be averaged over a current line, and random orientations yield the condition

$$
\overline{e^{ \pm i \varphi}}=0
$$

that is, the orientation of the magnetization of a local region has an azimuthal angle $\varphi$ with random values in the range 0 to $2 \pi$; therefore

$$
\overline{\xi_{ \pm \mp}}=0 \text {. }
$$

This randomness condition reduces the resistivity matrix to the simple form

$$
\overline{\left[\rho_{s r}(\mathbf{r})\right]}=C_{D}^{-1}\left(\begin{array}{cccc}
\overline{\xi_{++}} & 0 & 0 & 0 \\
0 & \overline{\xi_{--}} & 0 & 0 \\
0 & 0 & 0 & \overline{\xi_{0}} \\
0 & 0 & \overline{\xi_{0}} & 0
\end{array}\right)
$$


As a consequence, from the diagonal elements with respect to spin indices [i.e., for $(++)$ and $(--)$ ],

$$
\bar{E}=C_{D}^{-1} \overline{\xi_{++}} j_{++}=C_{D}^{-1} \overline{\xi_{--}} j_{--},
$$

and these expressions reduce the global conductance to the same value as for the homogeneous limit.

The above argument suggests that granular solids are magnetically self-averaging, due to randomness in the distribution of granules. However, in the local limit for granular solids, current lines do not necessarily sample all the scattering in the medium with equal weight; the relative weight depends upon the local resistivity differences. ${ }^{41}$ This exclusion does not in any way alter the conclusion that the system is magnetically self-averaging. In general, in the local limit, the average scattering includes that from the matrix and interfaces (interfaces are probed regardless of the relative values of the local resistivities) and only a fraction of the scattering in the granules, due to partial penetration of the current lines. The only difference between the two limiting cases is at most the contribution from the granules. Thus, the magnetoresistance does not depend exponentially on the average distance between adjacent granules and it does not vanish in the local limit. It is in this sense that granular solids are magnetically self-averaging.

\section{B. Magnetoresistance}

We now turn to a discussion of the magnetoresistance of magnetically self-averaging systems. Based on the hypothesis made in the previous section, the global conductivity for a given magnetic configuration $M$ of the system is the sum of the conductivities of the individual spin channels,

$$
\sigma^{M}=\sum_{\alpha} \sigma_{\alpha}^{M}=\sum_{\alpha}\left(\bar{\rho}_{\alpha}^{M}\right)^{-1} \propto \operatorname{Tr}\left(\bar{\Delta}_{M}^{-1}\right),
$$

where Tr stands for the trace in spin space. The dependence of the resistivity on the magnetic configuration $M$ is determined by $\bar{\Delta}$, Eq. (5.8). For antiferromagnetic and random configurations the average magnetization $\bar{M}$ is zero and $\bar{\Delta}(\bar{M}=0)=0$, while for the ferromagnetic configuration the magnetization $\bar{M}$ reaches its saturation value $M_{s}$, and $\bar{\Delta}\left(\bar{M}=M_{s}\right)=\bar{\Delta}(\hat{\mathbf{M}}(\mathbf{r})=\hat{\mathbf{H}})$ is given by Eq. (5.8) with $\hat{\mathbf{M}}(\mathbf{r})$ along the direction of the magnetic field $\mathbf{H}$. For any magnetically self-averaging system, i.e., magnetic granular systems and layered structures in the CPP geometry, the maximum magnetoresistance ratio, found by using Eqs. (5.7)-(5.26) and (6.6), is identical to that previously found for the CPP geometry, ${ }^{4}$ i.e.,

$$
R \equiv \frac{\rho(\bar{M}=0)-\rho\left(\bar{M}=M_{s}\right)}{\rho\left(\bar{M}=M_{s}\right)}=\frac{|\bar{\Delta}|^{2}}{\Delta_{0}^{2}-|\bar{\Delta}|^{2}} .
$$

Equation (6.7) shows that, although the mean free path is not a relevant length scale for the MR of magnetically self-averaging systems, it nevertheless enters the expression for MR through the average scattering $\bar{\Delta}$, since it sets the scale for the resistivity.

Until now, the giant MR (GMR) effect has been ob- served mostly in multilayered structures for the CIP geometry for which the resistivity $\bar{\rho}_{\alpha}^{M}$ depends exponentially on the thickness of the layers relative to the mean free paths [that is, $\bar{\rho}_{\alpha}^{M}$ is not given by Eq. (5.26)], and MR vanishes in the local limit. The fact that this is not a magnetically self-averaging configuration has led to the erroneous impression that the GMR effect intrinsically depends on the dimensions of the magnetic and nonmagnetic components relative to the mean free path.

The actual magnetoresistance ratio $R$ will be smaller because there are spin-diffusion processes inherent in magnetic granular films that reduce the spin accumulation between regions of different magnetization. As we have shown elsewhere for layered structures, ${ }^{8}$ regions of different magnetization that are normal (perpendicular) to the applied electric field produce spin diffusion which reduces the magnetoresistance ratio $R$. Similarly, for granular films the large differences in resistivities for the zero field and fully aligned magnetic configurations predicted in Eq. (6.4) will be reduced by spin-diffusion processes not taken into account in our hypothesis that the current density and resistivity are uncorrelated. In addition, one can create mixing of currents in the spin channels by spin-flip processes, for example, by electronmagnon interactions at higher temperatures, in which case our analysis has to be $\operatorname{modified},{ }^{7}$ or in the presence of magnetic domains. Finally, it is conceivable that magnetic granular films grown epitaxially might not be magnetically self-averaging, either due to the shape of the granules or the patterned way in which they could be deposited. ${ }^{42-45}$

\section{DISCUSSION AND SUMMARY}

Electron transport or conduction in inhomogeneous solids is a venerable topic; until recently the focus was on nonmagnetic media. For this case there is charge accumulation due to local differences in the dielectric constants (band structure) and scattering rates of the conduction electrons; this produces local fields that vary spatially, even in the presence of a uniformly applied external field. In magnetic materials that are locally inhomogeneous there is spin accumulation in addition to the charge accumulation that is attendant on electron transport. This arises from a combination of spin-dependent band structures and spin-dependent scattering rates that vary from one region to another. In normal conduction processes the incoming current is unpolarized with respect to spin; however, due to the spin dependence of the medium, the current develops a spin dependence; i.e., one is dealing with spin-polarized transport. When the polarization takes place outside the medium whose conductivity is being studied, one calls this spin injection, e.g., the bipolar spin switch that was recently proposed by Johnson. ${ }^{46}$ The formalism we have derived is equally applicable to normal and spin-polarized transport in magnetic materials.

Earlier formulations of our theory have been confined to magnetic structures that are collinearly aligned (lo- 
cally ferromagnetic or antiferromagnetic). Indeed, in this special case the currents and fields (effective) are diagonal in the spin indices referring to the conduction electrons. In this paper we have developed a theory of electron transport in inhomogeneous magnetic structures that are oriented noncollinearly and/or randomly; as we have shown it is necessary to introduce spinor currents and effective spinor fields (off diagonal in spin space) to describe this conduction.

For current in the plane of the layers (presumed to be homogeneous) the electric field is a constant and, therefore, from the external boundary conditions, which are independent of spin, the global (measured) conductivity is readily given in terms of the two-point conductivities, Eq. (4.9). In this case, CIP, there is neither spin nor charge accumulation as we have assumed the layers to be homogeneous. We have recently relaxed this assumption and considered the effects of magnetic-domain formation on transport in multilayers. ${ }^{8}$ Under the more general conditions there is spin accumulation in CIP as well as CPP, so that the field $\mathbf{E}_{\gamma \delta}$, Eq. (2.13), is spin dependent for both CIP and CPP.

Under the most general conditions, one obtains the global (measured) conductivity from the two-point conductivity only after one has found the field or current distributions by viewing the constitutive relation, Eq. (2.15), as an integral equation. For certain simplifying geometries, one knows the fields or currents and can circumvent solving this integral equation. Also, for magnetically self-averaging conditions (Sec. VI A) the global conductivity is readily found without solving the integral equation; due to randomness, the resistivity and current density are not correlated and one can average over them separately; see Eq. (6.1). Under these conditions, which are satisfied as long as one neglects the spin diffusion inherent in granular solids, the resistivity and MR are given directly in terms of the average scattering encountered by the conduction electrons, $\bar{\Delta}$, Eq. (5.6). For the CPP geometry of magnetic multilayers, the same results are obtained in an exact way, due to the symmetries associated with current conduction in this geometry; see Eq. (5.37). A prediction of our model that is just a straightforward consequence of this self-averaging property is that the resistivity and magnetoresistance of magnetically self-averaging systems are independent of the local details of the scattering strength distribution and of the magnetization orientation.

While our formulation of spin transport in inhomogeneous media has not stressed the role of spin accumulation and diffusion we have demonstrated in Ref. 31 that our internal electric field, Eq. (2.13), can be written in terms of spin-dependent chemical potentials which satisfy the same diffusion equation that is used in the quasiclassical Boltzmann-equation approach developed by Valet and Fert. ${ }^{7}$ Finally, we stress that when one neglects spinflip processes, spin accumulation is a maximum; therefore, it should not be erroneously concluded that this accumulation has been overlooked in our treatment. ${ }^{27}$

\section{ACKNOWLEDGMENT}

This research was supported by Office of Naval Research Grant No. N00014-91-J-1695.

\section{APPENDIX A: PROPERTIES OF SPINOR INTERNAL FIELDS, GENERALIZED CURRENTS, AND CONDUCTIVITIES}

The change of a quantization axis arises from a coordinate transformation from an "old" reference frame to a "new" one. This amounts to a rotation in threedimensional space, under which all physical quantities must transform geometrically, through their corresponding linear representations of the group $\mathrm{SU}(2)$.

This rotation can be characterized by the spherical polar angles $(\theta, \varphi)$ subtended by the "old" axis $z$ with respect to the "new" one $z^{\prime}$ (see Fig. 2). The polar angle $\theta$ is the angle between the axes $z$ and $z^{\prime}$, and the azimuthal angle $\varphi$ is the one subtended with respect to the $x^{\prime}$ axis by the projection of $z$ onto the $x^{\prime}-y^{\prime}$ plane.

For spinor field operators associated with spin one-half, the corresponding matrix representation of the rotation group is the $2 \times 2$ unitary matrix

$$
\begin{aligned}
C & =R_{\mathbf{n}}(\phi)=\exp \left[-i \frac{\phi}{2}(\hat{\boldsymbol{\sigma}} \cdot \mathbf{n})\right] \\
& =\cos (\phi / 2)-i(\hat{\boldsymbol{\sigma}} \cdot \mathbf{n}) \sin (\phi / 2),
\end{aligned}
$$

which is a rotation through an angle $\phi$ in the direction of $\mathbf{n}$ and where $\hat{\boldsymbol{\sigma}}$ stands for the vector set of Pauli matrices. Let us now calculate the matrix associated with the required change of quantization axis through polar angles $(\theta, \varphi)$. We can visualize this rotation as taking the $z$ axis onto the $z^{\prime}$ axis through the angle $\phi=\theta$; thus, the corresponding rotational axis lies on the $x^{\prime}-y^{\prime}$ plane, and being itself perpendicular to the projection onto the $x^{\prime}-y^{\prime}$ plane of the $z$ axis, it is characterized by the unit vector $\mathbf{n}$ with polar angles $\theta_{\mathbf{n}}=\pi / 2$ and $\varphi_{\mathbf{n}}=\varphi-\pi / 2$ (see Fig. 2); therefore,

$$
\begin{aligned}
C= & \cos (\theta / 2)-i\left[\sigma_{x} \cos \left(\varphi-\frac{\pi}{2}\right)\right. \\
& \left.+\sigma_{y} \sin \left(\varphi-\frac{\pi}{2}\right)\right] \sin (\theta / 2) \\
= & \left(\begin{array}{cc}
\cos (\theta / 2) & \sin (\theta / 2) e^{-i \varphi} \\
-\sin (\theta / 2) e^{i \varphi} & \cos (\theta / 2)
\end{array}\right) .
\end{aligned}
$$

For example, if the "old" axis $z$ is that which diagonalizes the local magnetization $\hat{\mathbf{M}}$, the polar angles $(\theta, \varphi)$ are the ones subtended by this magnetization with respect

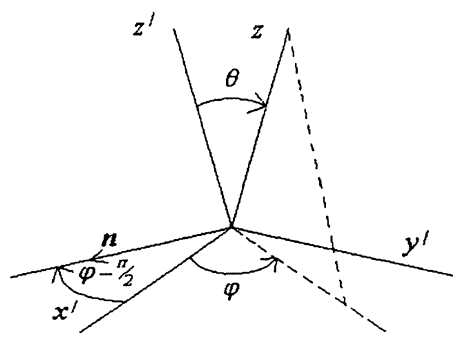

FIG. 2. Relative orientation of two sets of axes corresponding to two arbitrary choices of the quantization axis: $z$ is the "old" and $z^{\prime}$ the "new" quantization axis. 
to an arbitrary quantization axis $z^{\prime}$, and $C$ is the corresponding rotation matrix, which transforms all locally diagonalized quantities into their forms for an arbitrary axis of quantization.

Under the rotation described above, the spin- $1 / 2$ field operators transform according to the rules ("passive transformation")

$$
\Psi^{\prime \alpha}(\mathbf{r})=\left(C^{-1}\right)_{\beta}^{\alpha} \Psi^{\beta}(\mathbf{r})
$$

that is, using matrix notation,

$$
\Psi^{\prime}=C^{-1} \Psi
$$

(contravariant rule) and

$$
\Psi_{\alpha}^{\prime \dagger}(\mathbf{r})=(C)_{\alpha}^{\beta} \Psi_{\beta}^{\dagger}(\mathbf{r})
$$

that is,

$$
\Psi^{\dagger \dagger}=\Psi^{\dagger} C
$$

(covariant rule).

Correspondingly, the current operators

$$
\hat{\boldsymbol{j}}_{\alpha}^{\beta}(\mathbf{r})=\frac{e \hbar}{m i} \Psi_{\alpha}^{\dagger}(\mathbf{r}) \stackrel{\leftrightarrow}{\nabla}_{\mathbf{r}} \Psi^{\beta}(\mathbf{r})
$$

whose transformation properties are the same as for the product $\Psi_{\alpha}^{\dagger}(\mathbf{r}) \Psi^{\beta}(\mathbf{r})$, transform according to

$$
\hat{\boldsymbol{j}}_{\alpha}^{\prime}{ }_{\alpha}^{\beta}(\mathbf{r})=\left(C^{-1}\right)_{\delta}^{\beta} \hat{\mathbf{j}}_{\gamma}^{\delta}(\mathbf{r})(C)_{\alpha}^{\gamma}
$$

thus, the same is true for their expectation values,

$$
\mathbf{j}_{\alpha}^{\prime}{ }^{\beta}(\mathbf{r})=\left(C^{-1}\right)_{\delta}^{\beta} \mathbf{j}_{\gamma}^{\delta}(\mathbf{r})(C)_{\alpha}^{\gamma} .
$$

Therefore, the generalized current densities $\mathbf{j}_{\alpha}{ }^{\beta}(\mathbf{r})$ constitute a spinor of rank 2 , once covariant and once contravariant. In particular, they exhibit the following spinor-index symmetry:

$$
\left(\mathbf{j}_{\alpha}^{\beta}\right)^{*}(\mathbf{r})=\mathbf{j}_{\beta}^{\alpha}(\mathbf{r})
$$

which follows from $\left(\hat{\mathbf{j}}^{\dagger}\right)_{\alpha}^{\beta}=\hat{\mathbf{j}}_{\beta}^{\alpha}$ (adjoint operators, as follows from their definitions).

Similarly, the spin-dependent $2 \times 2$ scattering matrices $\Delta(\mathbf{r}), \xi(\mathbf{r}), \xi\left(\mathbf{r}, \mathbf{r}^{\prime}\right)$ have the same spinor character as the Pauli spin operator $\hat{\boldsymbol{\sigma}}$; that is, they are spinors of rank 2, once contravariant and once covariant; explicitly, they are $\Delta_{\beta}^{\alpha}(\mathbf{r}), \xi_{\beta}^{\alpha}(\mathbf{r}), \xi_{\beta}^{\alpha}\left(\mathbf{r}, \mathbf{r}^{\prime}\right)$. For example,

$$
\Delta_{\beta}^{\prime \alpha}(\mathbf{r})=\left(C^{-1}\right)_{\gamma}^{\alpha} \Delta_{\delta}^{\gamma}(\mathbf{r})(C)_{\beta}^{\delta} .
$$

It should be noticed that the scattering matrices are Hermitian in spin space; that is, they exhibit the spinor symmetries

$$
\left[\xi_{\beta}^{\alpha}(\mathbf{r})\right]^{*}=\xi_{\alpha}^{\beta}(\mathbf{r})
$$

and

$$
\left[\xi_{\beta}^{\alpha}\left(\mathbf{r}, \mathbf{r}^{\prime}\right)\right]^{*}=\xi_{\alpha}^{\beta}\left(\mathbf{r}, \mathbf{r}^{\prime}\right) ;
$$

this follows from their definition in terms of the scattering $T$ matrix, and can be explicitly verified from their form

$$
\begin{aligned}
{\left[\xi^{\alpha}{ }_{\boldsymbol{\beta}}(\mathbf{r})\right] } & \equiv\left(\begin{array}{ll}
\xi_{++}(\mathbf{r}) & \xi_{+-}(\mathbf{r}) \\
\xi_{-+}(\mathbf{r}) & \xi_{--}(\mathbf{r})
\end{array}\right) \\
& =\xi_{0}(\mathbf{r})+\hat{\boldsymbol{\sigma}} \cdot \boldsymbol{\xi}(\mathbf{r})=\left(\begin{array}{cc}
\xi_{0}(\mathbf{r})+|\boldsymbol{\xi}(\mathbf{r})| \cos \theta & |\boldsymbol{\xi}(\mathbf{r})| \sin \theta e^{-i \varphi} \\
|\boldsymbol{\xi}(\mathbf{r})| \sin \theta e^{i \varphi} & \xi_{0}(\mathbf{r})-|\boldsymbol{\xi}(\mathbf{r})| \cos \theta
\end{array}\right),
\end{aligned}
$$

which leads to Eqs. (5.33) and (5.34). Equation (A14) shows that the local scattering matrices are diagonalized when $\theta=0$ and $\varphi=0$, and the corresponding scattering values $\xi_{0}(\mathbf{r}) \pm|\boldsymbol{\xi}(\mathbf{r})|$ are real, confirming that Eq. (A12) holds for an arbitrary axis.

The Green's function $\mathcal{G}\left(\mathbf{r}, \mathbf{r}^{\prime}\right)$ has the same spinor character as the scattering matrices, as it is a matrix $\mathcal{G}^{\alpha}{ }_{\beta}\left(\mathbf{r}, \mathbf{r}^{\prime}\right)$, such that

$$
\Delta_{\beta}^{\alpha}(\mathbf{r}) \mathcal{G}_{\gamma}^{\beta}\left(\mathbf{r}, \mathbf{r}^{\prime}\right) \propto \delta_{\gamma}^{\alpha},
$$

in such a way that Eq. (3.4) is satisfied. Another way of finding the spinor character of the Green's function is through its definition as the expectation value of the product of quantum-mechanical field operators, that is,

$$
\mathcal{G}_{\beta}^{\alpha}\left(\mathbf{r}, \mathbf{r}^{\prime}\right) \propto\left\langle\Psi^{\alpha}(\mathbf{r}) \Psi_{\beta}^{\dagger}\left(\mathbf{r}^{\prime}\right)\right\rangle .
$$

The Green's function satisfies the spinor symmetry $(2.18)$, that is,

$$
\left[\left(\mathcal{G}^{\text {ret }}\right)_{\beta}^{\alpha}\left(\mathbf{r}, \mathbf{r}^{\prime}\right)\right]^{*}=\left(\mathcal{G}^{\text {adv }}\right)_{\alpha}^{\beta}\left(\mathbf{r}^{\prime}, \mathbf{r}\right) \text {. }
$$

The transport content of the Green's function is in the "path-ordered transport exponential," Eq. (3.16), which, as we show in Appendix D, is Hermitian in position-spin space, that is,

$$
\left[\mathcal{P}_{\beta}^{\alpha}\left(\mathbf{r}, \mathbf{r}^{\prime}\right)\right]^{*}=\mathcal{P}_{\alpha}^{\beta}\left(\mathbf{r}^{\prime}, \mathbf{r}\right)
$$

as a consequence of the Hermiticity of the scattering matrices, Eq. (A12).

On the other hand, the two-point conductivity $\boldsymbol{\sigma}\left(\mathbf{r}, \mathbf{r}^{\prime}\right)$, being proportional to the current-current correlation $\boldsymbol{\sigma}\left(\mathbf{r}, \mathbf{r}^{\prime}\right) \propto\left\langle\left[\hat{\mathbf{j}}(\mathbf{r}), \hat{\mathbf{j}}\left(\mathbf{r}^{\prime}\right)\right]\right\rangle$, is a fourth-rank spinor, twice covariant and twice contravariant: $\boldsymbol{\sigma}_{\alpha}{ }^{\beta},{ }_{\gamma}{ }^{\delta}\left(\mathbf{r}, \mathbf{r}^{\prime}\right)$; explicitly,

$$
\boldsymbol{\sigma}_{\alpha}{ }^{\beta},{ }_{\gamma}{ }^{\delta}\left(\mathbf{r}, \mathbf{r}^{\prime}\right) \propto\left\langle\left[\hat{\mathbf{j}}_{\alpha}{ }^{\beta}(\mathbf{r}), \hat{\mathbf{j}}_{\gamma}{ }^{\delta}\left(\mathbf{r}^{\prime}\right)\right]\right\rangle
$$

or, in terms of the Green's function,

$$
\boldsymbol{\sigma}_{\alpha}^{\beta},{ }_{\gamma}^{\delta}\left(\mathbf{r}, \mathbf{r}^{\prime}\right) \propto \mathcal{G}_{\gamma}^{\beta}\left(\mathbf{r}, \mathbf{r}^{\prime}\right) \mathcal{G}_{\alpha}^{\delta}\left(\mathbf{r}^{\prime}, \mathbf{r}\right) .
$$

Therefore, its transformation rule is

$$
\begin{aligned}
\boldsymbol{\sigma}^{\prime}{ }_{\alpha}^{\beta},{ }_{\gamma}^{\delta}\left(\mathbf{r}, \mathbf{r}^{\prime}\right) \\
\quad=\left(C^{-1}\right)_{\lambda}^{\beta}\left(C^{-1}\right)_{\nu}^{\delta} \sigma_{\varepsilon}{ }^{\lambda},{ }_{\mu}{ }^{\nu}\left(\mathbf{r}, \mathbf{r}^{\prime}\right)(C)_{\alpha}^{\varepsilon}(C)_{\gamma}^{\mu} .
\end{aligned}
$$

On the other hand, the generalization of Maxwell electric fields are "internal fields" that, in accordance with Eq. (2.13), should have the same spinor character as the vertex function, which, by inspection of Eqs. (2.10) and 
(2.11), is a spinor matrix with spinor character identical to that of the scattering matrices, that is,

$$
\mathbf{E}_{\beta}^{\alpha} \propto \Gamma_{\beta}^{\alpha} .
$$

Thus, the internal fields transform according to

$$
\mathbf{E}^{\prime \alpha}{ }_{\beta}(\mathbf{r})=\left(C^{-1}\right)_{\gamma}^{\alpha} \mathbf{E}_{\delta}^{\gamma}(\mathbf{r})(C)_{\beta}^{\delta},
$$

i.e., like a spinor of rank 2 , once covariant and once contravariant. This is consistent with the linear constitutive relationship, Eq. (2.15), relating the currents, Eq. (A9), to the internal fields, Eq. (A23), namely,

$$
\mathbf{j}_{\alpha}{ }^{\beta}(\mathbf{r})=\int d^{3} r^{\prime} \boldsymbol{\sigma}_{\alpha}{ }^{\beta},{ }_{\gamma}{ }^{\delta}\left(\mathbf{r}, \mathbf{r}^{\prime}\right) \cdot \mathbf{E}_{\delta}^{\gamma}\left(\mathbf{r}^{\prime}\right) .
$$

In agreement with their definition in terms of a vertex function, the internal fields should satisfy

$$
\left[\mathbf{E}^{\alpha}{ }_{\beta}(\mathbf{r})\right]^{*}=\mathbf{E}_{\alpha}^{\beta}(\mathbf{r}) \text {. }
$$

The two-point conductivity tensor exhibits the following spinor-index symmetries:

$$
\left[\boldsymbol{\sigma}_{\alpha}^{\beta},{ }_{\gamma}^{\delta}\left(\mathbf{r}, \mathbf{r}^{\prime}\right)\right]^{*}=\boldsymbol{\sigma}_{\beta}^{\alpha},{ }_{\delta}^{\gamma}\left(\mathbf{r}, \mathbf{r}^{\prime}\right)
$$

and

$$
\boldsymbol{\sigma}_{\alpha}^{\beta},{ }_{\gamma}^{\delta}\left(\mathbf{r}, \mathbf{r}^{\prime}\right)=\boldsymbol{\sigma}_{\gamma}{ }^{\delta},{ }_{\alpha}^{\beta}\left(\mathbf{r}^{\prime}, \mathbf{r}\right),
$$

both of which follow from the symmetries of the currents [Eq. (A10)] or of the Green's functions [Eq. (A17)]. In particular, Eq. (A26) expresses the consistency of the linear constitutive relationship, Eq. (A24), with the symmetry properties of currents, Eq. (A10), and fields, Eq. (A25), and Eq. (A27) stands for the Onsager relations. ${ }^{47}$

Equation (A24) can be inverted by defining a two-point resistivity $\rho_{\beta}^{\alpha},{ }_{\delta}^{\gamma}\left(\mathbf{r}, \mathbf{r}^{\prime}\right)$, such that

$$
\begin{aligned}
\int d^{3} r^{\prime \prime} \boldsymbol{\rho}_{\beta}^{\alpha},{ }_{\delta}^{\gamma}\left(\mathbf{r}, \mathbf{r}^{\prime \prime}\right) \cdot \boldsymbol{\sigma}_{\gamma}{ }^{\delta},{ }_{\varepsilon}^{\lambda}\left(\mathbf{r}^{\prime \prime}, \mathbf{r}^{\prime}\right) \\
=\mathbb{1}_{3} \delta_{\varepsilon}^{\alpha} \delta_{\beta}^{\lambda} \delta\left(\mathbf{r}-\mathbf{r}^{\prime}\right)
\end{aligned}
$$

whence

$$
\mathbf{E}_{\beta}^{\alpha}(\mathbf{r})=\int d^{3} r^{\prime} \boldsymbol{\rho}_{\beta}^{\alpha},{ }_{\delta}\left(\mathbf{r}, \mathbf{r}^{\prime}\right) \cdot \mathbf{j}_{\gamma}^{\delta}\left(\mathbf{r}^{\prime}\right) .
$$

The two-point resistivity matrix transforms according to

$$
\begin{aligned}
& \boldsymbol{\rho}^{\prime \alpha}{ }_{\beta},{ }_{\delta}^{\gamma}\left(\mathbf{r}, \mathbf{r}^{\prime}\right) \\
& \quad=\left(C^{-1}\right)_{\epsilon}^{\alpha}\left(C^{-1}\right)_{\mu}^{\gamma} \rho_{\lambda}^{\varepsilon},{ }_{\nu}\left(\mathbf{r}, \mathbf{r}^{\prime}\right)(C)_{\beta}^{\lambda}(C)_{\delta}^{\nu} .
\end{aligned}
$$

It proves useful to introduce the "bi-index" notation of Sec. V A, $s=(\alpha \beta)$; to be more precise, we should now make the distinction between the covariant arrangement

$$
M^{s} \equiv M_{\beta}^{\alpha}
$$

and the contravariant arrangement

$$
M_{s} \equiv M_{\alpha}{ }^{\beta},
$$

where $M$ is an arbitrary quantity. For example, we can rewrite Eq. (A24) in the form

$$
\mathbf{j}_{s}(\mathbf{r})=\int d^{3} r^{\prime} \boldsymbol{\sigma}_{s r}\left(\mathbf{r}, \mathbf{r}^{\prime}\right) \cdot \mathbf{E}^{r}\left(\mathbf{r}^{\prime}\right)
$$

where the currents $\mathbf{j}_{s}(\mathbf{r})$ become $1 \times 4$ "row" matrices, the fields $\mathbf{E}^{s}(\mathbf{r})$ become $4 \times 1$ "column" matrices, and the two-point conductivity $\sigma_{s r}\left(\mathbf{r}, \mathbf{r}^{\prime}\right)$ becomes a $4 \times 4$ matrix; similarly, we can rewrite Eq. (A29) in the form

$$
\mathbf{E}^{s}(\mathbf{r})=\int d^{3} r^{\prime} \boldsymbol{\rho}^{s r}\left(\mathbf{r}, \mathbf{r}^{\prime}\right) \cdot \mathbf{j}_{r}\left(\mathbf{r}^{\prime}\right)
$$

The transformation rules for the two-point conductivity and resistivity can be rewritten

$$
\boldsymbol{\sigma}^{\prime}{ }_{r s}\left(\mathbf{r}, \mathbf{r}^{\prime}\right)=\sigma_{m n}\left(\mathbf{r}, \mathbf{r}^{\prime}\right)(D)_{r}^{m}(D)_{s}^{n}
$$

and

$$
\boldsymbol{\rho}^{\prime r s}\left(\mathbf{r}, \mathbf{r}^{\prime}\right)=\left(D^{-1}\right)_{m}^{r}\left(D^{-1}\right)_{n}^{s} \boldsymbol{\rho}^{m n}\left(\mathbf{r}, \mathbf{r}^{\prime}\right),
$$

where the effective transformation matrices are

$$
(D)_{r}^{m}=(C)_{\alpha}^{\varepsilon}\left(C^{-1}\right)_{\lambda}^{\beta}
$$

and $D^{-1}$ is the inverse of the matrix $D$. The elements of the matrix (A37) can be explicitly evaluated by using Eq. (A2), whence

$$
D=\left(\begin{array}{cccc}
c^{2} & s^{2} & c s e^{i \varphi} & c s e^{-i \varphi} \\
s^{2} & c^{2} & -c s e^{i \varphi} & -c s e^{-i \varphi} \\
-c s e^{-i \varphi} & c s e^{-i \varphi} & c^{2} & -s^{2} e^{-2 i \varphi} \\
-c s e^{i \varphi} & c s e^{i \varphi} & -s^{2} e^{2 i \varphi} & c^{2}
\end{array}\right)
$$

where $c=\cos (\theta / 2)$ and $s=\cos (\theta / 2)$.

In this appendix, as well as in Appendix E, we make a careful distinction between covariant and contravariant indices, as this is essential every time that a change in quantization axis is performed. In the main text, however, we have preferred to keep the notation as light as possible, and avoided such notational subtleties.

\section{APPENDIX B: ALTERNATIVE COVARIANT FORMALISM}

The currents are uniquely defined by Eqs. (2.7) and (2.8) or by the requirement that they constitute a secondrank spinor with diagonal physical currents for any choice of a quantization axis.

On the other hand, the internal fields might be regarded as defined solely by the constitutive relation, Eq. (2.15), and devoid of a more fundamental meaning. Even though this is not quite so, as we showed in the text by defining the fields by absorbing the vertex corrections, we can still explore the degree of ambiguity involved in such relaxed definition.

Let us consider, for instance, as an alternative constitutive relation [as a replacement for Eq. (2.15)] 


$$
\mathbf{j}_{\alpha \beta}(\mathbf{r})=\int d^{3} \mathbf{r}^{\prime} \boldsymbol{\sigma}_{\alpha \gamma}\left(\mathbf{r}, \mathbf{r}^{\prime}\right) \tilde{\mathbf{E}}_{\gamma \beta}\left(\mathbf{r}^{\prime}\right)
$$

where $\sigma_{\alpha \gamma}\left(\mathbf{r}, \mathbf{r}^{\prime}\right)$ is the bubble part of the current-current correlation of the spinor current with the total current, which can be expressed in terms of spectral functions as $\boldsymbol{\sigma}_{\alpha \beta}\left(\mathbf{r}, \mathbf{r}^{\prime}\right)=\frac{4}{\pi} \frac{e^{2}}{\hbar}\left(\frac{\hbar^{2}}{2 m}\right)^{2} A_{\beta \delta}\left(\mathbf{r}, \mathbf{r}^{\prime}\right) \stackrel{\leftrightarrow}{\nabla}_{\mathbf{r}} \overleftrightarrow{\nabla}_{\mathbf{r}^{\prime}} A_{\delta \alpha}\left(\mathbf{r}^{\prime}, \mathbf{r}\right)$

where summation over repeated indices is implied. The effective field is then defined as

$$
\tilde{\mathbf{E}}_{\gamma \beta}\left(\mathbf{r}^{\prime}\right)=\mathbf{E}\left(\mathbf{r}^{\prime}\right) \delta_{\gamma \beta}+\int d^{3} r_{1} d^{3} r_{2} \boldsymbol{\rho}_{\gamma \delta}\left(\mathbf{r}^{\prime}, \mathbf{r}_{1}\right) \cdot \boldsymbol{\sigma}_{\delta \beta}^{\prime}\left(\mathbf{r}_{1}, \mathbf{r}_{2}\right) \cdot \mathbf{E}\left(\mathbf{r}_{2}\right)
$$

where $\boldsymbol{\rho}_{\gamma} \delta$ is the inverse of the bubble part of the conductivity $\sigma_{\gamma \delta}$ and $\sigma^{\prime}{ }_{\delta \beta}$ is the difference between the full local conductivity and the bubble contribution. The effective field of Eq. (2.13), introduced in conjunction with the fourth-rank spinor conductivity, is defined in terms of the vertex function $\Gamma_{\alpha \beta}(234)$, while the field associated with the second-rank spinor conductivity, Eq. (B3), is defined in terms of the long-range part of the conductivity $\boldsymbol{\sigma}_{\delta \beta}^{\prime}$ and of the inverse of the short-range (bubble) part of the conductivity, $\boldsymbol{\rho}_{\delta \boldsymbol{\beta}}$.

While the constitutive relation of Eq. (B1) may look simpler than that of Eq. (2.15), the complications are hidden in the effective field of Eq. (B3).

\section{APPENDIX C: DIVERGENCE OF THE GENERALIZED CURRENTS}

In this appendix we show that the generalized current is divergenceless for the case of our scattering potential, Eq. (2.6). tions

In effect, the spinor field operators satisfy the equa-

$$
-i \hbar \frac{\partial}{\partial t} \Psi_{\alpha}^{\dagger}=-\frac{\hbar^{2}}{2 m} \nabla^{2} \Psi_{\alpha}^{\dagger}+\Psi_{\gamma}^{\dagger} V_{\gamma \alpha}
$$

as well as

$$
i \hbar \frac{\partial}{\partial t} \Psi_{\beta}=-\frac{\hbar^{2}}{2 m} \nabla^{2} \Psi_{\beta}+V_{\beta \gamma} \Psi_{\gamma}
$$

where $V_{\gamma \alpha}$ are the spinor components of the scattering potential, Eq. (2.6). In Eq. (C2) we have used the fact that the potential is self-adjoint, $V^{\dagger}=V$. Multiplication of Eq. (C1) by $\Psi_{\beta}$ and of Eq. (C2) by $\Psi_{\alpha}^{\dagger}$, subtraction of the resultant equations, and evaluation of the corresponding expectation value yields

$$
\boldsymbol{\nabla} \cdot \mathbf{j}_{\alpha \beta}+\frac{\partial \rho_{\alpha \beta}}{\partial t}=w_{\alpha \beta}
$$

where $\mathbf{j}_{\alpha \beta}$ is the current density, defined by Eqs. (2.7) and (2.8),

$$
\rho_{\alpha \beta}=\left\langle e \Psi_{\alpha}^{\dagger}(\mathbf{r}) \Psi_{\beta}(\mathbf{r})\right\rangle
$$

is the charge density, and $w_{\alpha \beta}$ is the expectation value,

$$
w_{\alpha \beta}(\mathbf{r})=-\left\langle\frac{e}{i \hbar}\left[\Psi(\mathbf{r}) \Psi^{\dagger}(\mathbf{r}), V(\mathbf{r})\right]_{\beta \alpha}\right\rangle .
$$

On the other hand, $\Psi(\mathbf{r}) \Psi^{\dagger}(\mathbf{r})$ is essentially the equaltime and equal-position Green's function, which is determined by the self-energy $\Sigma(\mathbf{r})$. For our scattering potential, Eq. (2.6), we assumed that $\hat{\mathbf{M}}_{a}$ is a classical vector; therefore, $[\Sigma(\mathbf{r}), V(\mathbf{r})]=0$. In fact, this conclusion can be understood physically in that our rigid impurity moments $\hat{\mathbf{M}}_{a}$ are unable to cause spin flips.

As a consequence, in the steady state, where $\rho_{\alpha \beta}$ is independent of time, we arrive from Eq. (C3) at the condition (2.21), i.e., that the spinor current density is divergenceless.

\section{APPENDIX D: PROPERTIES OF THE PATH-ORDERING OPERATOR}

The path-ordering operator $P_{\mathbf{r}^{\prime} \rightarrow \mathbf{r}}$ reorders the noncommuting $2 \times 2$ spin scattering matrices appearing in any product, along the straight path $\Gamma\left[\mathbf{r}, \mathbf{r}^{\prime}\right]$ that starts at point $\mathbf{r}^{\prime}$ and ends up at point $\mathbf{r}$, and from right to left.

In other words, if we introduce a length parameter $s$ along the straight path $\Gamma\left[\mathbf{r}, \mathbf{r}^{\prime}\right]$, starting at point $\mathbf{r}^{\prime}$ (with $s\left[\mathbf{r}^{\prime}\right]=0$ ), that is, if

$$
s(\mathbf{r})=R\left(\mathbf{r}-\mathbf{r}^{\prime}\right)
$$

then

$$
\begin{aligned}
& P_{\mathbf{r}^{\prime} \rightarrow \mathbf{r}}\left[\Delta\left(\mathbf{r}_{1}\right) \Delta\left(\mathbf{r}_{2}\right) \cdots \Delta\left(\mathbf{r}_{N}\right)\right] \\
& \quad=\Delta\left(\mathbf{r}\left(s_{\sigma_{N}}\right)\right) \Delta\left(\mathbf{r}\left(s_{\sigma_{N-1}}\right)\right) \cdots \Delta\left(\mathbf{r}\left(s_{\sigma_{1}}\right)\right)
\end{aligned}
$$

where $\mathbf{r}_{j}=\mathbf{r}\left(s_{j}\right)$ and $\left(\sigma_{1}, \ldots, \sigma_{N}\right)$ is a permutation of $(1, \ldots, N)$, such that

$$
s_{\sigma_{1}}<s_{\sigma_{2}}<\cdots<s_{\sigma_{N}} .
$$

This path ordering is the analog (for spatially varying quantities) of the time ordering used in quantum field theory and many-body theory; ${ }^{48}$ a similar path ordering is actually used (in a four-dimensional space) in the discussion of non-Abelian gauge theories. ${ }^{49}$

The following symmetry property relates the two opposite ways of ordering matrices along a path:

$$
\begin{aligned}
\left\{P_{\mathbf{r}^{\prime} \rightarrow \mathbf{r}}\right. & {\left.\left[\Delta\left(\mathbf{r}_{1}\right) \Delta\left(\mathbf{r}_{2}\right) \cdots \Delta\left(\mathbf{r}_{N}\right)\right]\right\}^{T} } \\
& =P_{\mathbf{r} \rightarrow \mathbf{r}^{\prime}}\left\{\left[\Delta\left(\mathbf{r}_{1}\right)\right]^{T}\left[\Delta\left(\mathbf{r}_{2}\right)\right]^{T} \cdots\left[\Delta\left(\mathbf{r}_{N}\right)\right]^{T}\right\},
\end{aligned}
$$


where the superscript $T$ stands for the matrix transpose; Eq. (D4) follows from the reversal of the matrix product under transposition.

In the context of transport in inhomogeneous magnetic structures, path ordering arises in the process of solving the differential equation (3.4), when $k^{2}(\mathbf{r})$ is a spin matrix that corresponds to varying orientations for the corresponding magnetization vectors (noncollinear magnetizations $)$. By introducing a function $\mathcal{F}\left(\mathbf{r}, \mathbf{r}^{\prime}\right)$,

$$
\mathcal{G}\left(\mathbf{r}, \mathbf{r}^{\prime}\right) \equiv-\frac{1}{4 \pi R} \mathcal{F}\left(\mathbf{r}, \mathbf{r}^{\prime}\right)
$$

and by placing it into Eq. (3.5) one obtains the firstorder differential equation for $\mathcal{F}\left(\mathbf{r}, \mathbf{r}^{\prime}\right)$ with the "initial condition" $\mathcal{F}(\mathbf{r}, \mathbf{r})=1$,

$$
\left[\frac{\partial}{\partial s}-i k(\mathbf{r}(s))\right] \mathcal{F}\left(\mathbf{r}(\mathbf{s}), \mathbf{r}^{\prime}\right)=0
$$

where we have introduced the length parameter of Eq. (D1). Then, integrating both sides of Eq. (D6) and using the "initial condition" we get

$$
\begin{aligned}
\mathcal{F}\left(\mathbf{r}, \mathbf{r}^{\prime}\right) & =1+\int_{0}^{s} d s^{\prime \prime} \frac{\partial \mathcal{F}\left(\mathbf{r}\left(s^{\prime \prime}\right), \mathbf{r}^{\prime}\right)}{\partial s^{\prime \prime}} \\
& =1+\int_{\Gamma\left[\mathbf{r}, \mathbf{r}^{\prime}\right]} d s^{\prime \prime} i k\left(\mathbf{r}\left(s^{\prime \prime}\right)\right) \mathcal{F}\left(\mathbf{r}\left(s^{\prime \prime}\right), \mathbf{r}^{\prime}\right)
\end{aligned}
$$

Iteration of Eq. (D7) yields the series expansion

$$
\mathcal{F}\left(\mathbf{r}, \mathbf{r}^{\prime}\right)=1+\int_{\Gamma\left[\mathbf{r}, \mathbf{r}^{\prime}\right]} d s_{1} i k\left(\mathbf{r}_{1}\right)+\int_{\Gamma\left[\mathbf{r}, \mathbf{r}^{\prime}\right]} d s_{2} i k\left(\mathbf{r}_{2}\right) \int_{\Gamma\left[\mathbf{r}_{2}, \mathbf{r}^{\prime}\right]} d s_{1} i k\left(\mathbf{r}_{1}\right)+\cdots
$$

Using the identity

$$
P_{\mathbf{r}^{\prime} \rightarrow \mathbf{r}}\left\{\left[\int_{\Gamma\left[\mathbf{r}, \mathbf{r}^{\prime}\right]} d s^{\prime \prime} i k\left(\mathbf{r}\left(s^{\prime \prime}\right)\right)\right]^{n}\right\}=n ! \int_{\Gamma\left[\mathbf{r}, \mathbf{r}^{\prime}\right]} d s_{n} i k\left(\mathbf{r}_{n}\right) \int_{\Gamma\left[\mathbf{r}_{n}, \mathbf{r}^{\prime}\right]} d s_{n-1} i k\left(\mathbf{r}_{n-1}\right) \cdots \int_{\Gamma\left[\mathbf{r}_{2}, \mathbf{r}^{\prime}\right]} d s_{1} i k\left(\mathbf{r}_{1}\right)
$$

we can rewrite the Dyson series (D8) in a path-ordered form ${ }^{49}$

$$
\mathcal{F}\left(\mathbf{r}, \mathbf{r}^{\prime}\right)=\sum_{n=0}^{\infty} \frac{1}{n !} P\left\{\left[\int_{\Gamma\left[\mathbf{r}, \mathbf{r}^{\prime}\right]} d s^{\prime \prime} i k\left(\mathbf{r}\left(s^{\prime \prime}\right)\right)\right]^{n}\right\}
$$

As the sum "inside" this time-ordered product is the power-series expansion of the exponential function, this Dyson series can be recast into an "exponential form"

$$
\mathcal{F}\left(\mathbf{r}, \mathbf{r}^{\prime}\right)=P_{\mathbf{r}^{\prime} \rightarrow \mathbf{r}} \exp \left[i \int_{\Gamma\left[\mathbf{r}, \mathbf{r}^{\prime}\right]} d s^{\prime \prime} k\left(\mathbf{r}^{\prime \prime}\right)\right] .
$$

The path-ordering operator in Eq. (D11) takes care of the noncommutativity of the spin matrices, rearranging them automatically from the right in the direction from $\mathbf{r}^{\prime}$ to $\mathbf{r}$ (increasing arc length $s$ ). Thus, Eq. (D11) is a shorthand for the infinite series of Eq. (D8). In particular, it follows straightforwardly that Eq. (D8) is a solution of Eq. (D6). Therefore, from Eqs. (D5) and (D11), the Green's function solution, Eq. (3.7), follows. i.e.,

Finally, the path-ordered function (D11) is Hermitian,

$$
\left[\mathcal{F}_{\beta}^{\alpha}\left(\mathbf{r}, \mathbf{r}^{\prime}\right)\right]^{*}=\mathcal{F}_{\alpha}^{\beta}\left(\mathbf{r}^{\prime}, \mathbf{r}\right)
$$

and it leads to the corresponding Hermitian symmetry of Eq. (A18). These conclusions follow from the symmetry of the path-ordering operator, Eq. (D4), and from the Hermitian symmetry of the scattering matrices, Eq. (A12).

\section{APPENDIX E: INVERSION}

\section{OF SPINOR MATRICES IN THE LOCAL LIMIT}

The two-point conductivity is, in general, proportional to the product of two Green's functions. The correspond- ing spin-index structure is completely contained in the path-ordered transport exponential

$$
\mathcal{P}_{\beta}^{\alpha}\left(\mathbf{r}, \mathbf{r}^{\prime}\right)=\left\{P_{\mathbf{r}^{\prime} \rightarrow \mathbf{r}} \exp \left[-\frac{1}{2} \xi\left(\mathbf{r}, \mathbf{r}^{\prime}\right) R\right]\right\}_{\beta}^{\alpha},
$$

where $R=\left|\mathbf{r}-\mathbf{r}^{\prime}\right|$.

In the local limit, as $\mathbf{r} \rightarrow \mathbf{r}^{\prime}$, the path-ordered transport exponential becomes

$$
e_{\beta}^{\alpha}\left(\mathbf{r}, \mathbf{r}^{\prime}\right)=\left\{\exp \left[-\frac{1}{2} \xi(\mathbf{r}) R\right]\right\}_{\beta}^{\alpha}
$$

where $\xi(\mathbf{r})$ is the local reciprocal mean free path matrix [Eq. (3.9)] and path ordering has become superfluous as all matrices commute locally within one region.

These matrices can be evaluated by means of the identity $^{50}$

$$
\begin{aligned}
f(a+\mathbf{b} \cdot \hat{\boldsymbol{\sigma}})= & \frac{1}{2}[f(a+b)+f(a-b)] \mathbb{1} \\
& +\frac{\mathbf{b}}{2 b}[f(a+b)-f(a-b)] \cdot \hat{\boldsymbol{\sigma}},
\end{aligned}
$$

where $\mathbb{1}$ is the unit $2 \times 2$ matrix, whence

$$
\begin{aligned}
e^{\alpha}{ }_{\beta}= & \left(e^{-R \xi / 2}\right)_{\beta}^{\alpha} \\
= & \left(e^{-R \xi_{0} / 2}\right)\left[\cosh \left(\frac{R|\boldsymbol{\xi}|}{2}\right) \mathbb{1}-\hat{\boldsymbol{\sigma}} \cdot \hat{\boldsymbol{\xi}} \sinh \left(\frac{R|\boldsymbol{\xi}|}{2}\right)\right]_{\beta}^{\alpha} \\
= & \left(e^{-R \xi_{0} / 2}\right)\left[\delta_{\beta}^{\alpha} \cosh \left(\frac{R|\boldsymbol{\xi}|}{2}\right)\right. \\
& \left.-(\hat{\boldsymbol{\sigma}} \cdot \hat{\boldsymbol{\xi}})_{\beta}^{\alpha} \sinh \left(\frac{R|\boldsymbol{\xi}|}{2}\right)\right],
\end{aligned}
$$

or, explicitly, using matrix notation, when the vector $\hat{\boldsymbol{\xi}}$ has polar angles $(\theta, \varphi)$ with respect to the chosen quantization axis, 


$$
\left[e_{\beta}^{\alpha}\right]=\left(e^{-R \xi_{0} / 2}\right)\left(\begin{array}{cc}
\cosh \left(\frac{R|\boldsymbol{\xi}|}{2}\right)-\sinh \left(\frac{R|\boldsymbol{\xi}|}{2}\right) \cos \theta & -\sinh \left(\frac{R|\boldsymbol{\xi}|}{2}\right) \sin \theta e^{-i \varphi} \\
-\sinh \left(\frac{R|\boldsymbol{\xi}|}{2}\right) \sin \theta e^{i \varphi} & \cosh \left(\frac{R|\boldsymbol{\xi}|}{2}\right)+\sinh \left(\frac{R|\boldsymbol{\xi}|}{2}\right) \cos \theta
\end{array}\right)
$$

The elements of the two-point conductivity are essentially given by

$$
\boldsymbol{\sigma}_{\alpha}^{\beta},{ }_{\gamma}^{\delta} \propto e_{\gamma}^{\beta} e_{\alpha}^{\delta} .
$$

Notice the symmetries

$$
\left(e_{\beta}^{\alpha}\right)^{*}=e_{\alpha}^{\beta},
$$

which are dictated by Eq. (A18).

By using the symmetries of the two-point conductivity, Eqs. (A26) and (A27), and setting $\mathbf{r} \rightarrow \mathbf{r}^{\prime}$ in the local limit, one concludes from Eq. (E6) that there are only seven independent conductivities, four of which are real:

$$
\begin{aligned}
& \boldsymbol{\sigma}_{+}^{+},{ }_{+}^{+} \propto\left(e^{+}\right)^{2} \in \Re \\
& \boldsymbol{\sigma}{ }_{-},{ }_{-}^{-} \propto\left(e^{-}\right)^{2} \in \Re, \\
& \boldsymbol{\sigma}_{+}{ }^{+},{ }_{-}^{-}=\boldsymbol{\sigma}{ }_{-}^{-},{ }_{+}^{+} \propto\left|e^{+}\right|^{2} \in \Re, \\
& \boldsymbol{\sigma}_{+}^{-},{ }_{-}^{+}=\boldsymbol{\sigma}{ }_{-}^{+},{ }_{+}^{-} \propto e^{+}+e^{-} \in \Re, \\
& \boldsymbol{\sigma}_{+}^{-},{ }_{+}^{-}=\left(\boldsymbol{\sigma}_{-}^{+},{ }_{-}^{+}\right)^{*} \propto\left(e^{-}\right)^{2}, \\
& \boldsymbol{\sigma}_{+}^{-},+^{+}=\left(\boldsymbol{\sigma}_{-}^{+},+^{+}\right)^{*} \\
& =\boldsymbol{\sigma}+{ }_{+}^{+},+^{-}=\left(\boldsymbol{\sigma}_{+}^{+},{ }_{-}^{+}\right)^{*} \propto e^{+}{ }_{+} e_{+}^{-}, \\
& \boldsymbol{\sigma}_{+}^{-},{ }_{-}^{-}=\left(\boldsymbol{\sigma}_{-}^{+},{ }_{-}^{-}\right)^{*} \\
& =\boldsymbol{\sigma}_{-}^{-},+^{-}=\left(\boldsymbol{\sigma}_{-}^{-},{ }_{-}^{+}\right)^{*} \propto e^{-} e^{-}{ }_{+}
\end{aligned}
$$

These elements can be computed in the local limit by using the limiting expressions

$$
\begin{gathered}
e^{-R \xi_{0}} \cosh (R|\boldsymbol{\xi}|) \rightarrow \delta(\mathbf{R}) \frac{\xi_{0}}{\xi_{0}^{2}-\boldsymbol{\xi}^{2}}, \\
e^{-R \xi_{0}} \sinh ^{2}\left(\frac{R|\boldsymbol{\xi}|}{2}\right) \rightarrow \delta(\mathbf{R}) \frac{\boldsymbol{\xi}^{2}}{2\left(\xi_{0}^{2}-\boldsymbol{\xi}^{2}\right) \xi_{0}} \\
e^{-R \xi_{0}} \sinh (R|\boldsymbol{\xi}|) \rightarrow \delta(\mathbf{R}) \frac{|\boldsymbol{\xi}|}{\xi_{0}^{2}-\boldsymbol{\xi}^{2}}
\end{gathered}
$$

Then, the limiting matrix, Eq. (5.32), follows.

Finally, we write the explicit reduced form that Eq. (5.32) takes for the "diagonalizing" choice $\theta=0, \varphi=$ 0 , namely,

$$
\left[\sigma^{r s}\right] \propto\left(\begin{array}{cccc}
\left(\xi_{0}+|\boldsymbol{\xi}|\right)^{-1} & 0 & 0 & 0 \\
0 & \left(\xi_{0}-|\boldsymbol{\xi}|\right)^{-1} & 0 & 0 \\
0 & 0 & 0 & \left(\xi_{0}\right)^{-1} \\
0 & 0 & \left(\xi_{0}\right)^{-1} & 0
\end{array}\right)
$$

Inverting Eq. (E8),

$$
\left[\rho^{r s}\right] \propto\left(\begin{array}{cccc}
\xi_{0}+|\boldsymbol{\xi}| & 0 & 0 & 0 \\
0 & \xi_{0}-|\boldsymbol{\xi}| & 0 & 0 \\
0 & 0 & 0 & \xi_{0} \\
0 & 0 & \xi_{0} & 0
\end{array}\right)
$$

and reverting to an arbitrary axis by means of the transformation rule (A36) and with Eq. (A38), the local resistivity, Eq. (5.36), follows.
${ }^{1}$ R. F. Carcia and A. Suna, J. Appl. Phys. 54, 2000 (1983).

${ }^{2}$ R. E. Camley and J. Barnás, Phys. Rev. Lett. 63, 664 (1989); J. Barnás, A. Fuss, R. E. Camley, P. Grunberg, and W. Zinn, Phys. Rev. B 42, 8110 (1990).

${ }^{3}$ S. Zhang, P. M. Levy, and A. Fert, Phys. Rev. B 45, 8689 (1992).

${ }^{4}$ S. Zhang and P. M. Levy, J. Appl. Phys. 69, 4786 (1991).

${ }^{5}$ H. E. Camblong and P. M. Levy, J. Appl. Phys. 73, 5533 (1993).

${ }^{6}$ H. E. Camblong, S. Zhang, and P. M. Levy, Phys. Rev. B 47, 4735 (1993).

7 T. Valet and A. Fert, J. Magn. Magn. Mater. 121, 378 (1993); Phys. Rev. B 48, 7099 (1993).

${ }^{8}$ S. Zhang and P. M. Levy, Phys. Rev. B 49, 6089 (1994).

${ }^{9}$ G. D. Mahan, Many-Particle Physics, 2nd ed. (Plenum, New York, 1990), Sec. 3.8 and Chap. 7.

${ }^{10}$ G. Rickayzen, Green's Functions and Condensed Matter (Academic Press, San Diego, 1980), Chap. 4.

${ }^{11} \mathrm{Z}$. Tešanović, M. V. Jaric, and S. Maekawa, Phys. Rev. Lett. 57, 2760 (1986).

${ }^{12}$ B. L. Johnson and R. E. Camley, Phys. Rev. B 44, 9997 (1991).

${ }^{13}$ P. M. Levy, Z.-P. Shi, S. Zhang, H. E. Camblong, and J.
L. Fry, J. Magn. Magn. Mater. 121, 357 (1993).

${ }^{14}$ M. B. Stearns, J. Magn. Magn. Mater. 104-107, 1745 (1992); A. C. Ehrlich, Phys. Rev. Lett. 71, 2300 (1993).

${ }^{15}$ R. Q. Hood and L. M. Falicov, Phys. Rev. B 46, 8287 (1992).

${ }^{16}$ P. B. Visscher and H. Zhang, J. Magn. Magn. Mater. 121, 449 (1993); Phys. Rev. B 48, 6672 (1993).

${ }^{17}$ A. Oguri, Y. Asano, and S. Maekawa, J. Phys. Soc. Jpn. 61, 2652 (1992); Y. Asano, A. Oguri, and S. Maekawa, Phys. Rev. B 48, 6192 (1993).

18 The magnetic multilayers studied to date have ratios $d / \lambda_{\operatorname{mfp}} \gg 1$, where $d$ is the thickness of the entire structure and $\lambda_{\mathrm{mfp}}$ is the elastic mean free path. Therefore the conduction is impurity dominated: The roughness of the outer surfaces does not dominate the resistivity and those quantum effects coming from confinement are not of paramount importance. If differences in the potentials between layers are sizable compared to the Fermi energy, one should use wave functions that are eigenstates of the multilayer potential, and then evaluate the scattering by using these wave functions. It would be incorrect to consider the quantum wells arising from potential differences between layers as confinement potentials. If indeed they confined electrons 
to wells, one would not have conduction perpendicular to the plane of the layers, which is inconsistent with the data on the systems studied to date in the CPP geometry.

19 J. Rammer, Rev. Mod. Phys. 63, 781 (1991).

${ }^{20}$ W. Kohn and J. M. Luttinger, Phys. Rev. 108, 590 (1957).

${ }^{21}$ R. H. Silsbee, Bull. Magn. Reson. 2, 284 (1980); M. Johnson and R. H. Silsbee, Phys. Rev. Lett. 55, 1790 (1985); Phys. Rev. B 35, 4959 (1987); 37, 5312 (1988); 37, 5326 (1988).

22 Spinor currents were introduced by J. Slonczewski, Phys. Rev. B 39, 995 (1989), to study transmission through tunneling barriers.

${ }^{23}$ This is an extension of the Kubo formula for the conductivity (see Rickayzen, Ref. 10) to include the spin variables.

${ }^{24}$ See G. D. Mahan, Many-Particle Physics, 1st ed. (Plenum, New York, 1981), pp. 205-208, and Rickayzen, Ref. 10, pp. 127-129, for a discussion of the difference between total and external electric fields.

${ }^{25}$ G. Baym and L. P. Kadanoff, Phys. Rev. 124, 287 (1961). They showed that the conservation laws for transport imply relationships between the vertex functions and one-electron propagators, and that it is necessary to respect them so that the vertex one uses satisfies the conservation laws.

${ }^{26}$ H. E. Camblong, S. Zhang, and P. M. Levy, J. Appl. Phys. 75, 6906 (1994).

${ }^{27}$ It has been incorrectly assumed [see M. A. M. Gijs, S. K. Lenczowski, and J. B. Giesbers, Phys. Rev. Lett. 70, 3343 (1993) and G. E. W. Bauer, Phys. Rev. Lett. 69, 1676 (1992)] that spin accumulation effects have been ignored in theories of the CPP MR which are based on Eq. (2.15), e.g., Refs. 4, 5, and 6, as well as in S. Zhang and P. M. Levy, Phys. Rev. B 47, 6776 (1993).

${ }^{28}$ P. C. van Son, H. van Kempen, and P. Wyder, Phys. Rev. Lett. 58, 2271 (1987).

${ }^{29}$ M. Johnson and R. H. Silsbee, Phys. Rev. Lett. 60, 377 (1988), and Ref. 21.

${ }^{30}$ H. E. Camblong, Phys. Rev. B 51, 1855 (1995).

${ }^{31}$ P. M. Levy, H. E. Camblong, and S. Zhang, J. Appl. Phys. 75, 7076 (1994).

${ }^{32}$ A. Fert, J. Phys. C 2, 1784 (1969); A. Fert and I. A. Campbell, J. Phys. F 6, 849 (1976); J. Phys. Paris Colloq. 32, C1-46 (1971); I. A. Campbell, A Fert, and O. Jaoul, J. Phys. C Suppl. 1, S95 (1970); O. Jaoul, I. A. Campbell, and A. Fert, J. Magn. Magn. Mater. 5, 23 (1977); A. Fert, Physica 86-88B, 491 (1977).

${ }^{33}$ C. L. Kane, R. A. Serota, and P. A. Lee, Phys. Rev. B 37, 6701 (1988).

${ }^{34}$ H. E. Camblong and P. M. Levy, Phys. Rev. Lett. 69, 2835 (1992); J. Magn. Magn. Mater. 121, 446 (1993).

${ }^{35}$ B. Dieny, A. Vedyayev, and N. Ryzhanova, J. Magn. Magn. Mater. 121, 366 (1993).

${ }^{36}$ P. M. Levy, K. Ounadjela, S. Zhang, Y. Wang, C. B. Sommers, and A. Fert, J. Appl. Phys. 67, 5914 (1990); P. M. Levy, S. Zhang, and A. Fert, Phys. Rev. Lett. 65, 1643 (1990).

37 A. Vedyayev, B. Dieny, and N. Ryzhanova, Europhys. Lett. 19, 329 (1992).

${ }^{38}$ B. Dieny, J. P. Nozieres, V. S. Speriosu, B. A. Gurney, and
D. R. Wilhoit, Appl. Phys. Lett. 61, 2111 (1992).

${ }^{39}$ As resistivity is proportional to the scattering of conduction electrons, when one takes the average of the resistivities as in Eq. (??), one is, at some level, taking the average of the scattering; therefore, it seems intuitively plausible that it is proportional to the average of the scattering and does not depend on how it is spatially distributed.

${ }^{40}$ This result was originally predicted by Zhang and Levy (Ref. 4), and was later confirmed experimentally. For example, see W. P. Pratt, Jr., S.-F. Lee, J. M. Slaughter, R. Loloee, P. A. Schroeder, and J. Bass, Phys. Rev. Lett. 66, 3060 (1991); W. P. Pratt, Jr., S.-F. Lee, Q. Yang, D. Holody, R. Loloee, P. A. Schroeder, and J. Bass, J. Appl. Phys. 73, 5326 (1993), J. Magn. Magn. Mater. 126, 406 (1993); P. A. Schroeder, J. Bass, P. Holody, S.-F. Lee, R. Loloee, W. P. Pratt, Jr., and Q. Yang, in Magnetism and Structure in Systems of Reduced Dimension, edited by R. F. C. Farrow et al., Vol. 309 of NATO Advanced Study Institute, Series B: Physics (Plenum Press, New York, 1993), p. 129.

${ }^{41}$ Only if the current lines do not undergo significant refraction at the interfaces, namely, when the relative differences in local conductivities are small, can one safely assume that the global resistivity is proportional to the average scattering in the whole system. Instead, if the local resistivity in the granules is much larger than that of the matrix and the mean free path is much smaller than the size of the granules, the current lines tend to avoid the granules and only partial penetration into the granules occurs. Therefore, in the limit of very large relative differences in local conductivities, the global resistivity again becomes an average scattering, but one which includes only contributions from the matrix and the interfaces.

${ }^{42}$ S. S. P. Parkin, R. F. C. Farrow, T. A. Rabedeau, R. F. Marks, G. R. Harp, Q. H. Lam, C. Chappert, M. Toney, R. Savoy, and R. Geiss, Europhys. Lett. 22, 455 (1993); T. A. Rabedeau, M. Toney, R. F. Marks, S. S. P. Parkin, R. F. C. Farrow, and G. Harp, Phys. Rev. B 48, 16810 (1993); see also Y. Fujii, in Metallic Superlattices, edited by T. Shinjo and T. Takada (Elsevier, Amsterdam, 1987) Chap. 2, p. 33.

${ }^{43}$ T. L. Hylton, K. R. Coffey, M. A. Parker, and J. K. Howard, Science 261, 1021 (1993).

${ }^{44}$ B. Rodmacq, G. Palembo, and P. Gerard, J. Magn. Magn. Mater. 118, L11 (1993).

${ }^{45}$ M. Parker, J. Barnard, D. Seale, M. Tan, S. Hossain, and H. Fujiwara (unpublished).

${ }^{46}$ M. Johnson, Science 260, 320 (1993); Appl. Phys. Lett. 63, 1435 (1993).

${ }^{47}$ L. D. Landau and E. M. Lifshitz, Statistical Physics (Pergamon Press, Oxford, 1980), Pt. 1, p. 366.

${ }^{48}$ See, for example, Ref. 9, pp. 83-87, or G. Baym, Lectures on Quantum Mechanics (Addison-Wesley, Redwood City, CA, 1990), pp. 142-145.

${ }^{49}$ See, for example, K. Huang, Quarks, Leptons, and Gauge Fields (World Scientific, Singapore, 1982), pp. 74-76.

${ }^{50}$ L. D. Landau and E. M. Lifshitz, Quantum Mechanics (Pergamon Press, Oxford, 1977), p. 204. 